\title{
A POSTERIORI ERROR ANALYSIS FOR A CONTINUOUS SPACE-TIME FINITE ELEMENT METHOD FOR A HYPERBOLIC INTEGRO-DIFFERENTIAL EQUATION
}

\author{
FARDIN SAEDPANAH
}

\begin{abstract}
An integro-differential equation of hyperbolic type, with mixed boundary conditions, is considered. A continuous space-time finite element method of degree one is formulated. A posteriori error representations based on space-time cells is presented such that it can be used for adaptive strategies based on dual weighted residual methods. A posteriori error estimates based on weighted global projections and local projections are also proved.
\end{abstract}

\section{Introduction}

We study the initial-boundary value problem (we use '.' to denote the time derivative),

$$
\begin{array}{ll}
\ddot{u}(x, t)-\nabla \cdot \sigma(u ; x, t)=f(x, t) & \text { in } \Omega \times(0, T), \\
u(x, t)=0 & \text { on } \Gamma_{\mathrm{D}} \times(0, T), \\
\sigma(u ; x, t) \cdot n=g(x, t) & \text { on } \Gamma_{\mathrm{N}} \times(0, T), \\
u(x, 0)=u^{0}(x), \quad \dot{u}(x, 0)=v^{0}(x) & \text { in } \Omega,
\end{array}
$$

that is a hyperbolic type integro-differential equation arising, e.g., in modeling dynamic fractional/linear viscoelasticity. Here $u$ is the displacement vector, $f$ and $g$ represent, respectively, the volume and surface loads. The stress $\sigma=\sigma(u ; x, t)$ is determined by

$$
\sigma(t)=\sigma_{0}(t)-\int_{0}^{t} \mathcal{K}(t-s) \sigma_{0}(s) d s,
$$

with

$$
\sigma_{0}(t)=2 \mu_{0} \epsilon(t)+\lambda_{0} \operatorname{Tr}(\epsilon(t)) \mathrm{I}
$$

where I is the identity operator, $\epsilon$ is the strain which is defined by $\epsilon=\frac{1}{2}(\nabla u+$ $\left.(\nabla u)^{T}\right)$, and $\mu_{0}, \lambda_{0}>0$ are elastic constants of Lamé type. The kernel $\mathcal{K}$ is considered to be either smooth (exponential), or no worse than weakly singular, so in both cases with the properties that

$$
\mathcal{K} \geq 0, \quad \mathcal{K}^{\prime} \leq 0, \quad\|\mathcal{K}\|_{L_{1}\left(\mathbb{R}^{+}\right)}=\kappa<1 .
$$

For examples of problems of this type see, e.g., 11] and [17] and their references.

Date: November 14, 2012.

1991 Mathematics Subject Classification. 65M60, 45K05.

Key words and phrases. integro-differential equation, continuous Galerkin finite element method, convolution kernel, stability, a posteriori estimate. 
We note that, e.g., completely monotone functions, i.e., functions $b \in L_{1}(0, \infty) \cap$ $\mathcal{C}^{2}(0, \infty)$, such that

$$
(-1)^{k} D_{t}^{k} b(t) \geq 0, \quad t \in(0, \infty), k=0,1,2,
$$

satisfy (1.2), when $\|b\|_{L_{1}\left(\mathbb{R}^{+}\right)}<1$. Hence, Mittag-Leffler type kernels, that are weakly singular and arise in fractional order viscoelasticity, satisfy (1.2), see, e.g., 11.

Existence, uniqueness and regularity of solution of a problem of the form (1.1) has been studied in [18. A posteriori analysis of temporal finite element approximation of a parabolic type problem and discontinuous Galerkin finite element approximation of a quasi-static $(\ddot{u} \approx 0)$ linear viscoelasticity problem has been studied, respectively, in [3] and [21. For analysis and numerical solution of integro-differential equations and related problems, from the extensive literature, we mention [3], 11, [14, 17, and their references.

Here we consider a continuous space-time finite element approximation of degree one, $c G(1) c G(1)$, for problem (1.1). A similar method has been applied to the second order hyperbolic problems in [5], where the main objective is goal-oriented adaptive discretization of the problems. We introduce a posteriori error representations, that can be a basis for goal-oriented adaptive strategies based on dual weighted residual (DWR) method, see [5] and [6] for details and computational aspects on the DWR method. For examples of application of the DWR approach for problems in solid mechanics see [10, [20, and the references therein.

To evaluate the a posteriori error representations we need information about the continuous dual solution. Such information has to be obtained either through a priori analysis in form of bounds for the dual solution in certain Sobolev norms or through computation by solving the dual problem numerically. In this context we consider the former case and provide information through a priori analysis. To this end, we present two a posteriori error estimates based on global and local projections. A weighted global a posteriori error estimate is obtained, using global $L_{2}$-projections, for which the main framework is adapted from [8], and as an example of its specific application we refer to [9]. For the second a posteriori error estimate, that is based on local projections, we refer to [5].

The model problem (1.1) is of hyperbolic type, and therefore our analysis are based on special treatment of discretization of the wave equation perturbed with a memory term. Here we only present the theory.

The memory term causes the growing amount of data that has to be stored and used in each time step. The most commonly used algorithms for this integration are based on Lubich convolution quadrature 12 for fractional order operators, see also 19] for an improved version. For examples of the application of this approch to overcome the problem with the growing amount of data, that has to be stored and used in time stepping methods, see [1, 2], 3], 13, and [15. In particular see, e.g., 1 where sparse quadrature together with appropriate a posteriori error representaions and estimates have been used in adaptive strategies based on the DWR approach. We plan to address this issue together with numerical adaptation methods such as DWR approach in future work. However, we should note that this is not an issue for exponentially decaying memory kernels, in linear viscoelasticity, that are represented as a Prony series. In this case recurrence relationships can be derived which means recurrence formula are used for history updating, see [10] and 21] for more details. 
The outline of this paper is as follows. In $\S 2$ we define a weak form of (1.1) and the corresponding dual (adjoint) problem. In $\S 3$ we formulate a continuous Galerkin method of degree one. Then in $\S 4$ we present a posteriori error representations, from which, in $\S 5$ we prove a weighted global a posteriori error estimate. A localized a posteriori error estimate is also proved in $\S 6$. We remark on special cases regarding the memory term and regularity of the convolution kernel, when it is needed.

\section{Weak formulation and stability}

We let $\Omega \subset \mathbb{R}^{d}, d=2,3$, be a bounded polygonal domain with boundary $\Gamma=$ $\Gamma_{\mathrm{D}} \cup \Gamma_{\mathrm{N}}$ where $\Gamma_{\mathrm{D}}$ and $\Gamma_{\mathrm{N}}$ are disjoint and meas $\left(\Gamma_{\mathrm{D}}\right) \neq 0$. We introduce the function spaces $H=L_{2}(\Omega)^{d}, H_{\Gamma_{\mathrm{N}}}=L_{2}\left(\Gamma_{\mathrm{N}}\right)^{d}$, and $V=\left\{v \in H^{1}(\Omega)^{d}:\left.v\right|_{\Gamma_{\mathrm{D}}}=0\right\}$. We denote the norms in $H$ and $H_{\Gamma_{\mathrm{N}}}$ by $\|\cdot\|$ and $\|\cdot\|_{\Gamma_{\mathrm{N}}}$, respectively.

2.1. Weak formulation. We define a bilinear form (with the usual summation convention)

$$
a(v, w)=\int_{\Omega}\left(2 \mu_{0} \epsilon_{i j}(v) \epsilon_{i j}(w)+\lambda_{0} \epsilon_{i i}(v) \epsilon_{j j}(w)\right) d x, \quad v, w \in V,
$$

which is coercive on $V$, and we equip $V$ with the inner product $a(\cdot, \cdot)$ and norm $\|v\|_{V}^{2}=a(v, v)$. We define $A u=-\nabla \cdot \sigma_{0}(u)$, which is a selfadjoint, positive definite, unbounded linear operator, with $\mathcal{D}(A)=H^{2}(\Omega)^{d} \cap V$, and we use the norms $\|v\|_{s}=$ $\left\|A^{s / 2} v\right\|$.

We use a "velocity-displacement" formulation of (1.1) which is obtained by introducing a new velocity variable. Henceforth we use the new variables $u_{1}=u, u_{2}=\dot{u}$ and $u=\left(u_{1}, u_{2}\right)$ the pair of vector valued functions. Then the variational form is to find $u_{1}(t), u_{2}(t) \in V$ such that $u_{1}(0)=u^{0}, u_{2}(0)=v^{0}$, and

$$
\begin{aligned}
& \left(\dot{u}_{1}(t), v_{1}\right)-\left(u_{2}(t), v_{1}\right)=0, \\
& \left(\dot{u}_{2}(t), v_{2}\right)+a\left(u_{1}(t), v_{2}\right)-\int_{0}^{t} \mathcal{K}(t-s) a\left(u_{1}(s), v_{2}\right) d s \\
& \quad=\left(f(t), v_{2}\right)+\left(g(t), v_{2}\right)_{\Gamma_{N}}, \quad \forall v_{1}, v_{2} \in V, t \in(0, T) .
\end{aligned}
$$

Now we define the bilinear and linear forms $B: \mathcal{U} \times \mathcal{V} \rightarrow \mathbb{R}$ and $L: \mathcal{V} \rightarrow \mathbb{R}$ by

$$
\begin{aligned}
B(u, v)= & \int_{0}^{T}\left\{\left(\dot{u}_{2}, v_{2}\right)+a\left(u_{1}, v_{2}\right)-\int_{0}^{t} \mathcal{K}(t-s) a\left(u_{1}(s), v_{2}\right) d s\right. \\
& \left.+\left(\dot{u}_{1}, v_{1}\right)-\left(u_{2}, v_{1}\right)\right\} d t+\left(u_{1}(0), v_{1}(0)\right)+\left(u_{2}(0), v_{2}(0)\right), \\
L(v)= & \int_{0}^{T}\left\{\left(f, v_{2}\right)+\left(g, v_{2}\right)_{\Gamma_{\mathrm{N}}}\right\} d t+\left(u^{0}, v_{1}(0)\right)+\left(v^{0}, v_{2}(0)\right),
\end{aligned}
$$

where

$$
\begin{aligned}
& \mathcal{U}=H^{1}(0, T ; V) \times H^{1}(0, T ; H) \\
& \mathcal{V}=\left\{v=\left(v_{1}, v_{2}\right): v \in L_{2}(0, T ; H) \times L_{2}(0, T ; V), v_{i} \text { right continuous in } t\right\}
\end{aligned}
$$

We note that if we change the order of the time integrals in the convolution term as well as changing the role of the variables $s, t$, we have the second variant of the 
bilinear form $B$, that is,

$$
\begin{aligned}
B(u, v)= & \int_{0}^{T}\left\{\left(\dot{u}_{2}, v_{2}\right)+a\left(u_{1}, v_{2}\right)-\int_{t}^{T} \mathcal{K}(s-t) a\left(u_{1}(t), v_{2}(s)\right) d s\right. \\
& \left.+\left(\dot{u}_{1}, v_{1}\right)-\left(u_{2}, v_{1}\right)\right\} d t+\left(u_{1}(0), v_{1}(0)\right)+\left(u_{2}(0), v_{2}(0)\right) .
\end{aligned}
$$

We use this variant of $B$ in $\S 4$ for a posteriori error analysis.

The weak form 2.2. can be writen as: find $u \in \mathcal{U}$ such that,

$$
B(u, v)=L(v), \quad \forall v \in \mathcal{V} .
$$

Here the definition of the velocity $u_{2}=\dot{u}_{1}$ is enforced in the $L_{2}$ sense, and the initial data are placed in the bilinear form in a weak sense. A variant is used in [11 where the velocity has been enforced in the $H^{1}$ sense, without placing the initial data in the bilinear form. We also note that the initial data are retained by the choice of the function space $\mathcal{V}$, that consists of right continuous functions with respect to time.

Our error analysis is based on the duality arguments, and therefore we formulate the dual form of (2.6). To this end, we define the bilinear and linear forms $B_{\tau}^{*}$ : $\mathcal{V}^{*} \times \mathcal{U}^{*} \rightarrow \mathbb{R}, L_{\tau}^{*}: \mathcal{V}^{*} \rightarrow \mathbb{R}$, for $\tau \in \mathbb{R}^{\geq 0}$, by

$$
\begin{aligned}
B_{\tau}^{*}(v, z)= & \int_{\tau}^{T}\left\{-\left(v_{1}, \dot{z}_{1}\right)+a\left(v_{1}, z_{2}\right)-\int_{t}^{T} \mathcal{K}(s-t) a\left(v_{1}, z_{2}(s)\right) d s\right. \\
& \left.-\left(v_{2}, \dot{z}_{2}\right)-\left(v_{2}, z_{1}\right)\right\} d t+\left(v_{1}(T), z_{1}(T)\right)+\left(v_{2}(T), z_{2}(T)\right), \\
L_{\tau}^{*}(v)= & \int_{\tau}^{T}\left\{\left(v_{1}, j_{1}\right)+\left(v_{2}, j_{2}\right)\right\} d t+\left(v_{1}(T), z_{1}^{T}\right)+\left(v_{2}(T), z_{2}^{T}\right),
\end{aligned}
$$

where $j_{1}, j_{2}$ and $z_{1}^{T}, z_{2}^{T}$ represent, respectively, the load terms and the initial data of the dual (adjoint) problem. In case of $\tau=0$, we use the notation $B^{*}, L^{*}$ for short. Here

$$
\begin{aligned}
& \mathcal{U}^{*}=H^{1}(0, T ; H) \times H^{1}(0, T ; V), \\
& \mathcal{V}^{*}=\left\{v=\left(v_{1}, v_{2}\right): v \in L_{2}(0, T ; V) \times L_{2}(0, T ; H), v_{i} \text { left continuous in } t\right\} .
\end{aligned}
$$

We note that, recalling (2.4), $\mathcal{U} \subset \mathcal{V}^{*}, \mathcal{U}^{*} \subset \mathcal{V}$.

We also note that $B^{*}$ is the adjoint form of $B$. Indeed, integrating by parts with respect to time in $B$, then changing the order of integrals in the convolution term as well as changing the role of the variables $s, t$, we have,

$$
B(u, v)=B^{*}(u, v), \quad \forall u \in \mathcal{U}, v \in \mathcal{U}^{*} .
$$

Hence, the variational form of the dual problem is to find $z \in \mathcal{U}^{*}$ such that,

$$
B^{*}(v, z)=L^{*}(v), \quad \forall v \in \mathcal{V}^{*},
$$

that is a weak formulation of

$$
\ddot{z}_{2}+A z_{2}-\int_{t}^{T} \mathcal{K}(s-t) A z_{2}(s) d s=j_{1}-\frac{\partial}{\partial t} j_{2},
$$

with initial data $z_{1}^{T}, z_{2}^{T}$, and function $j=\left(j_{1}, j_{2}\right)$ that is defined by $L^{*}(w)=$ $\int_{0}^{T}(w, j) d t$.

We note that, since $z$ is a solution of (2.10), we have

$$
z_{1}=-\dot{z}_{2}-j_{2} \text {. }
$$


2.2. Stability of the solution of the dual problem. We know that stability estimates and the corresponding anlysis for dual problem is similar to the primal problem, however with opposite time direction. Hence, having a smooth or weakly singular kernel with (1.2), we can obtain the stability estimates (2.13) from, e.g., [4] or 11] for the continuous dual solution. Here we state stability estimates for the continuous dual solution in the following lemma, and we omit the proof for short. We note that the stability constant in (2.13) does not depend on $t$, and Gronwall's lemma has not been used, see [4] and [11. See also [16], [17] and [21], where stability estimates have been represented, in which the stability factor depends on $t$, due to Gronwall's lemma.

Lemma 1. Let $z$ be the solution of the dual problem (2.10) with sufficiently smooth data $z_{1}^{T}, z_{2}^{T}, j_{1}, j_{2}$. Then, for some constant $C=C(\kappa)$, we have stability estimates

$$
\left\|z_{1}(t)\right\|_{l}+\left\|z_{2}(t)\right\|_{l+1} \leq C\left\{\left\|z_{1}^{T}\right\|_{l}+\left\|z_{2}^{T}\right\|_{l+1}+\int_{t}^{T}\left(\left\|j_{1}\right\|_{l}+\left\|j_{2}\right\|_{l+1}\right) d r\right\}
$$

We note that, if we set $j_{1}=j_{2}=0$ in (2.13) and recall (2.11)-(2.12), then for $r \in[t, T]$ and $l \in \mathbb{R}$, we have

$$
\begin{aligned}
\left\|\dot{z}_{1}(r)\right\|_{l-1} & =\left\|\ddot{z}_{2}(r)\right\|_{l-1}=\left\|-A z_{2}(r)+\int_{r}^{T} \mathcal{K}(s-r) A z_{2}(s) d s\right\|_{l-1} \\
& \leq\left\|A z_{2}(r)\right\|_{l-1}+\int_{r}^{T} \mathcal{K}(s-r)\left\|A z_{2}(s)\right\|_{l-1} d s,
\end{aligned}
$$

that, recalling $\|\mathcal{K}\|_{L_{1}(\mathbb{R})}=\kappa$, for some $C=C(\kappa)$ implies

$$
\left\|\dot{z}_{1}(r)\right\|_{l-1}=\left\|\ddot{z}_{2}(r)\right\|_{l-1} \leq(1+\kappa) \max _{r \leq s \leq T}\left\|A z_{2}(r)\right\|_{l-1} \leq(1+\kappa) \max _{r \leq s \leq T}\left\|z_{2}(r)\right\|_{l+1} .
$$

From this and (2.13) we conclude the stability inequality

$$
\left\|\dot{z}_{1}(t)\right\|_{l-1}+\left\|z_{1}(t)\right\|_{l}+\left\|z_{2}(t)\right\|_{l+1} \leq C\left\{\left\|z_{1}^{T}\right\|_{l}+\left\|z_{2}^{T}\right\|_{l+1}\right\} .
$$

\section{The continuous Galerkin method}

Here we formulate the continuous Galerkin method of order one, cG(1)cG(1), for the primary and dual problems (2.6) and (2.10).

3.1. The cG method. Let $0=t_{0}<t_{1}<\cdots<t_{n-1}<t_{n}<\cdots<t_{N}=T$ be a partition of the time interval $[0, T]$. To each discrete time level $t_{n}$ we associate a triangulation $\mathcal{T}_{h}^{n}$ of the polygonal domain $\Omega$ with the mesh function,

$$
h_{n}(x)=h_{K}=\operatorname{diam}(K), \quad x \in K, K \in \mathcal{T}_{h}^{n},
$$

and a finite element space $V_{h}^{n}$ consisting of continuous piecewise linear polynomials. For each time subinterval $I_{n}=\left(t_{n-1}, t_{n}\right)$ of length $k_{n}=t_{n}-t_{n-1}$, we define intermediate triangulaion $\overline{\mathcal{T}}_{h}^{n}$ which is composed of mutually finest meshes of the neighboring meshes $\mathcal{T}_{h}^{n}, \mathcal{T}_{h}^{n-1}$ defined at discrete time levels $t_{n}, t_{n-1}$, respectively. The mesh function $\bar{h}_{n}$ is then defined by

$$
\bar{h}_{n}(x)=\bar{h}_{K}=\operatorname{diam}(K), \quad x \in K, K \in \overline{\mathcal{T}}_{h}^{n} .
$$

Correspondingly, we define the finite element spaces $\bar{V}_{h}^{n}$ consisting of continuous piecewise linear polynomials. This construction is used in order to allow continuity in time of the trial functions when the meshes change with time. Hence we obtain a decomposition of each time slab $\Omega^{n}=\Omega \times I_{n}$ into space-time cells $K^{n}=K \times I_{n}, K \in$ 
$\overline{\mathcal{T}}_{h}^{n}$ (prisms, for example, in case of $\Omega \subset \mathbb{R}^{2}$ ). We note the difference between the mesh functions $h_{n}$ and $\bar{h}_{n}$, and this is important in our a posteriori error analysis. The trial and test function spaces for the discrete form are, respectively:

$$
\begin{gathered}
\mathcal{U}_{h k}=\left\{U=\left(U_{1}, U_{2}\right): U \text { continuous in } \Omega \times[0, T],\left.U(x, t)\right|_{I_{n}} \text { linear in } t,\right. \\
\left.U\left(\cdot, t_{n}\right) \in\left(V_{h}^{n}\right)^{2},\left.U(\cdot, t)\right|_{I_{n}} \in\left(\bar{V}_{h}^{n}\right)^{2}\right\} \\
\mathcal{V}_{h k}=\left\{V=\left(V_{1}, V_{2}\right): V(\cdot, t) \text { continuous in } \Omega,\left.V(\cdot, t)\right|_{I_{n}} \in\left(V_{h}^{n}\right)^{2},\right. \\
\left.\left.V(x, t)\right|_{I_{n}} \text { piecewise constant in } t\right\} .
\end{gathered}
$$

We note that global continuity of the trial functions in $\mathcal{U}_{h k}$ requires the use of 'hanging nodes' if the spatial mesh changes across a time level $t_{n}$. In these 'irregular' nodal points the unknowns are eliminated by interpolating values at neighboring 'regular' nodal points, see [5], 22] and the references therein for practical aspects. We allow one hanging node per edge or face. On the other hand, the test functions, which are allowed to be discontinuous in time, are defined in the time slabs $\Omega \times I_{n}$ on the spatial meshes corresponding to $t_{n}$. In this discretization trial functions have support in both time intervals $I_{n}$ and $I_{n+1}$ adjacent to time instants $t_{n}$. Therefore on each time interval $I_{n}$, the trial functions defined at time instant $t_{n-1}$ on a mesh $\mathcal{T}_{h}^{n-1}$ overlap with test functions defined at time instant $t_{n}$ on a mesh $\mathcal{T}_{h}^{n}$. Hence, to form space-time integrals of multiplication of trial functions and test functions, we need the 'union' of the two triangulations, that we have denoted by $\overline{\mathcal{T}}_{h}^{n}$. We also note that, due to the structure of the elements of $\overline{\mathcal{T}}_{h}^{n}$, computations are feasible with reasonable effort if the grids $\mathcal{T}_{h}^{n-1}$ and $\mathcal{T}_{h}^{n}$ are related. Hierarchically structured spatial meshes, where $\overline{\mathcal{T}}_{h}^{n}$ is the set of most refined cells from the two grids, reduces the substantial work caused by the mesh transfer from one time level to the next. More details and references on the practical implementation can be found in [5].

In the construction of $\mathcal{U}_{h k}$ and $\mathcal{V}_{h k}$ we have associated the triangulation $\mathcal{T}_{h}^{n}$ with discrete time levels instead of the time slabs $\Omega^{n}$, and in the interior of time slabs we let $U$ be from the union of the finite element spaces defined on the triangulations at the two adjacent time levels. Associating triangulation with time slabs instead of time levels would yield a variant scheme which includes jump terms due to discontinuity at discrete time leveles, when coarsening happens. This means that there are extra degrees of freedom that one might use suitable projections for transfering solution at the time levels $t_{n}$, see [11.

The continuous Galerkin method, based on the variational formulation (2.2), is to find $U \in \mathcal{U}_{h k}$ such that,

$$
B(U, V)=L(V), \quad \forall V \in \mathcal{V}_{h k}
$$

The Galerkin orthogonality, with $u=\left(u_{1}, u_{2}\right)$ being the exact solution of (2.2), is then,

$$
B(U-u, V)=0, \quad \forall V \in \mathcal{V}_{h k} .
$$


From (3.4) we can recover the time stepping scheme,

$$
\begin{aligned}
& \quad \int_{I_{n}}\left\{\left(\dot{U}_{1}, V_{1}\right)-\left(U_{2}, V_{1}\right)\right\} d t=0, \\
& \int_{I_{n}}\left\{\left(\dot{U}_{2}, V_{2}\right)+a\left(U_{1}, V_{2}\right)-\int_{0}^{t} \mathcal{K}(t-s) a\left(U_{1}(s), V_{2}(t)\right) d s\right\} d t \\
& \quad=\int_{I_{n}}\left\{\left(f, V_{2}\right) d t+\left(g, V_{2}\right)_{\Gamma_{\mathrm{N}}}\right\} d t, \quad \forall\left(V_{1}, V_{2}\right) \in \mathcal{V}_{h k}, \\
& U_{1}(0)=u_{h}^{0}, \quad U_{2}(0)=v_{h}^{0},
\end{aligned}
$$

for suitable choice of $u_{h}^{0}, v_{h}^{0} \in V_{h}^{0}$ as approximations of the initial data $u^{0}, v^{0}$.

We define the orthogonal projections $\mathcal{P}_{h, n}: H \rightarrow V_{h}^{n}$ and $\mathcal{P}_{k, n}: L_{2}\left(I_{n}\right)^{d} \rightarrow$ $\mathbb{P}_{0}^{d}\left(I_{n}\right)$, respectively, by

$$
\begin{aligned}
\left(\mathcal{P}_{h, n} v-v, \chi\right) & =0, \quad \forall v \in H, \chi \in V_{h}^{n}, \\
\int_{I_{n}}\left(\mathcal{P}_{k, n} v-v\right) \cdot \psi d t & =0, \quad \forall v \in L_{2}\left(I_{n}\right)^{d}, \psi \in \mathbb{P}_{0}^{d}\left(I_{n}\right),
\end{aligned}
$$

with $\mathbb{P}_{0}^{d}$ denoting the set of all vector-valued constant polynomials. Correspondingly, we define $\mathcal{P}_{h} v$ and $\mathcal{P}_{k} v$ for $t \in I_{n}(n=1, \cdots, N)$, by $\left(\mathcal{P}_{h} v\right)(t)=\mathcal{P}_{h, n} v(t)$ and $\mathcal{P}_{k} v=\mathcal{P}_{k, n}\left(\left.v\right|_{I_{n}}\right)$. We note that, as a natural choice, we can set the initial data in (3.6) as

$$
u_{h}^{0}=\mathcal{P}_{h} u^{0}, \quad v_{h}^{0}=\mathcal{P}_{h} v^{0} .
$$

We also note that, when we do not change the spatial mesh or just refine the spatial mesh from one time level to the next one, i.e., $V_{h}^{n-1} \subset V_{h}^{n}, n=1, \ldots, N$, then we have $\bar{V}_{h}^{n}=V_{h}^{n}$.

We introduce the linear operator $A_{n, r}: V_{h}^{r} \rightarrow V_{h}^{n}$ by

$$
a\left(v_{r}, w_{n}\right)=\left(A_{n, r} v_{r}, w_{n}\right), \quad \forall v_{r} \in V_{h}^{r}, w_{n} \in V_{h}^{n} .
$$

We set $A_{n}=A_{n, n}$, with discrete norms

$$
\left\|v_{n}\right\|_{h, l}=\left\|A_{n}^{l / 2} v_{n}\right\|=\sqrt{\left(v_{n}, A_{n}^{l} v_{n}\right)}, \quad v_{n} \in V_{h}^{n} \text { and } l \in \mathbb{R},
$$

and $A_{h}$ so that $A_{h} v=A_{n} v$ for $v \in V_{h}^{n}$. We use $\bar{A}_{h}$ when it acts on $\bar{V}_{h}^{n}$.

\section{A posteriori error estimation: error representation}

Having certain regularity on the data, i.e., initial data $u^{0}, v^{0}$ and the force terms $f, g$, there are still two types of limitation for higher global regularity of a weak solution of (1.1). One is due to the mixed Dirichlet-Neumann boundary condition. This type of boundary condition are natural in practice, and a pure Dirichlet boundary condition cannot be realistic in applications. Other limitation is the singularity of the convolution kernel $\mathcal{K}$. This means that even with the pure Dirichlet boundary condition, higher regularity of a weak solution is limited, see 11, 18, though with smoother kernels we can get higher regularity. These, and other general motivations such as no practical use of a priori error estimates, call for adaptive meshes based on a posteriori error analysis.

Here a space-time cellwise error representation is given. The main framework is adapted from [ㅈ, and a general linear goal functional $L^{*}(\cdot)$ is used. For an example of a global goal functional, see Example1. This error representation can be used for 
goal-oriented adaptive strategies based on dual weighted residual (DWR) method. For more details on dual weighted residual method and its practical aspects for differential equations, see [5], 6] and references therein.

In the following, we recall and define some notations for this section. We denote the space-time cells $K^{n}=K \times I_{n}$ and $\partial K^{n}=\partial K \times I_{n}$, for $K \in \overline{\mathcal{T}}_{h}^{n}$, and it should be noticed that $\partial K^{n}$ is not the boundary of $K^{n}$. Also for a simplex $K$, we define the inner products $(\cdot, \cdot)_{K^{n}}=\int_{I_{n}}(\cdot, \cdot)_{K} d t,(\cdot, \cdot)_{\partial K^{n}}=\int_{I_{n}}(\cdot, \cdot)_{\partial K} d t$, and the corresponding $L_{2}$-norms

$$
\begin{aligned}
& \|\cdot\|_{K^{n}}=\left(\int_{I_{n}}\|\cdot\|_{K}^{2} d t\right)^{1 / 2}=\left(\int_{I_{n}}\|\cdot\|_{L_{2}(K)}^{2} d t\right)^{1 / 2}, \\
& \|\cdot\|_{\partial K^{n}}=\left(\int_{I_{n}}\|\cdot\|_{\partial K}^{2} d t\right)^{1 / 2}=\left(\int_{I_{n}}\|\cdot\|_{L_{2}(\partial K)}^{2} d t\right)^{1 / 2} .
\end{aligned}
$$

We also denote

$$
(\mathcal{K} * v)^{j}(t)=\int_{t_{j-1}}^{t_{j} \wedge t} \mathcal{K}(t-s) v(s) d s .
$$

Throughout we use the usual notations $a \wedge b=\min \{a, b\}$ and $a \vee b=\max \{a, b\}$.

Now we present three a posteriori error representations, and we note that the second error representation, (4.3), is space-time cellwise.

Theorem 1. Let $u$ and $U$ be the solutions of (2.6) and (3.4), respectively, and $L^{*}(\cdot)$ be the linear functional defined in (2.7). Then, denoting the error $e=U-u$, we have the error representations

$$
\begin{gathered}
L^{*}(e)=\sum_{K \in \mathcal{T}_{h}^{0}} \Theta_{0, K}+\sum_{n=1}^{N} \sum_{K \in \overline{\mathcal{T}}_{h}^{n}} \sum_{i=1}^{4} \Theta_{i, K}^{n}+\sum_{n=1}^{N} \sum_{j=1}^{n} \sum_{K \in \overline{\mathcal{T}}_{h}^{j}} \Theta_{5, K}^{n, j}, \\
L^{*}(e)=\sum_{K \in \mathcal{T}_{h}^{0}} \Theta_{0, K}+\sum_{n=1}^{N} \sum_{K \in \overline{\mathcal{T}}_{h}^{n}} \sum_{i=1}^{5} \Theta_{i, K}^{n}, \\
L^{*}(e)=\sum_{K \in \mathcal{T}_{h}^{0}} \Theta_{0, K}+\sum_{n=1}^{N} \sum_{K \in \overline{\mathcal{T}}_{h}^{n}} \sum_{i=1}^{4} \Theta_{i, K}^{n}+\sum_{n=1}^{N} \sum_{j=n}^{N} \sum_{K \in \overline{\mathcal{T}}_{h}^{j}} \Theta_{5, K}^{N, j},
\end{gathered}
$$

where, with $z_{h k} \in \mathcal{V}_{h k}$ being an approximation of the dual solution $z$ and $E_{h k} z=$ $z_{h k}-z$ being the error operator,

$$
\begin{aligned}
\Theta_{0, K} & =\left(U_{1}(0)-u^{0}, E_{h k} z_{1}(0)\right)_{K}+\left(U_{2}(0)-v^{0}, E_{h k} z_{2}(0)\right)_{K}, \\
\Theta_{1, K}^{n} & =\left(\dot{U}_{1}-U_{2}, E_{h k} z_{1}\right)_{K^{n}}, \quad \Theta_{2, K}^{n}=\left(\dot{U}_{2}-f, E_{h k} z_{2}\right)_{K}, \\
\Theta_{3, K}^{n} & =\left(g_{d}-g, E_{h k} z_{2}\right)_{\partial K^{n}}, \quad \Theta_{4, K}^{n}=\left(r_{d}, E_{h k} z_{2}\right)_{\partial K^{n}}, \\
\Theta_{5, K}^{n, j} & =-\int_{I_{n}}\left(\left(\mathcal{K} * r_{d}\right)^{j}(t), E_{h k} z_{2}(t)\right)_{\partial K} d t, \\
\Theta_{5, K}^{n} & =-\left(r_{d}, \int_{t}^{T} \mathcal{K}(s-t) E_{h k} z_{2}(s) d s\right)_{\partial K^{n}} \\
\Theta_{5, K}^{N, j} & =-\int_{I_{n}}\left(r_{d}(t), \int_{t_{j-1} \vee t}^{t_{j}} \mathcal{K}(s-t) E_{h k} z_{2}(s) d s\right)_{\partial K} d t .
\end{aligned}
$$


Here $r_{d}$ are the residuals representing the jumps of the normal derivatives $\sigma_{0}\left(U_{1}\right) \cdot n$, which are determined by

$$
\left.r_{d}\right|_{\Gamma}= \begin{cases}-\frac{1}{2}\left[\sigma_{0}\left(U_{1}\right) \cdot n\right] & \text { if } \Gamma \subset \partial K \backslash \partial \Omega \\ 0 & \text { if } \Gamma \subset \partial \Omega\end{cases}
$$

and $g_{d}$ are the contribution from the Neumann boundary condition defined by

$$
\left.g_{d}\right|_{\Gamma}= \begin{cases}\sigma\left(U_{1}\right) \cdot n=\left(\sigma_{0}\left(U_{1}\right)-\int_{0}^{t} \mathcal{K}(t-s) \sigma_{0}\left(U_{1}(s)\right) d s\right) \cdot n & \text { if } \Gamma \subset \partial K \cap \Gamma_{N}, \\ 0 & \text { otherwise. }\end{cases}
$$

Proof. Using the identity (2.9) and the Galerkin orthogonality (3.5) we have,

$$
\begin{aligned}
L^{*}(e) & =B^{*}(e, z)=B(e, z)=B\left(e, E_{h k} z\right)=B\left(U, E_{h k} z\right)-B\left(u, E_{h k} z\right) \\
& =B\left(U, E_{h k} z\right)-L\left(E_{h k} z\right)=R\left(U ; E_{h k} z\right)
\end{aligned}
$$

where $R(U ; \cdot)$ is the residual of the Galerkin approximation $U$ as a functional on the solution space $\mathcal{U}^{*}$. Then, by the definition of $B$ and $L$ in (2.3), we have

$$
\begin{aligned}
L^{*}(e)= & \left(U_{1}(0)-u^{0}, E_{h k} z_{1}(0)\right)+\left(U_{2}(0)-v^{0}, E_{h k} z_{2}(0)\right) \\
& +\int_{0}^{T}\left\{\left(\dot{U}_{1}, E_{h k} z_{1}\right)-\left(U_{2}, E_{h k} z_{1}\right)+\left(\dot{U}_{2}, E_{h k} z_{2}\right)+a\left(U_{1}, E_{h k} z_{2}\right)\right. \\
& \left.-\int_{0}^{t} \mathcal{K}(t-s) a\left(U_{1}(s), E_{h k} z_{2}(t)\right) d s\right\} d t \\
& -\int_{0}^{T}\left\{\left(f, E_{h k} z_{2}\right)+\left(g, E_{h k} z_{2}\right)_{\Gamma_{\mathrm{N}}}\right\} d t .
\end{aligned}
$$

Now, by partial integration with respect to the space variable and recalling $r_{d}$ from (4.6), we obtain

$$
\begin{array}{rl}
\int_{0}^{T} & a\left(U_{1}, E_{h k} z_{2}\right) d t \\
& =\sum_{n=1}^{N} \int_{I_{n}} \sum_{K \in \overline{\mathcal{T}}_{h}^{n}} a\left(U_{1}, E_{h k} z_{2}\right)_{K} d t=\sum_{n=1}^{N} \int_{I_{n}} \sum_{K \in \overline{\mathcal{T}}_{h}^{n}}\left(\sigma_{0}\left(U_{1}\right) \cdot n, E_{h k} z_{2}\right)_{\partial K} d t \\
& =\sum_{n=1}^{N} \int_{I_{n}}\left\{\sum_{E \in \mathcal{E}_{I}^{n}}\left(-\left[\sigma_{0}\left(U_{1}\right) \cdot n\right], E_{h k} z_{2}\right)_{E}+\sum_{E \in \mathcal{E}_{\Gamma_{\mathrm{N}}}^{n}}\left(\sigma_{0}\left(U_{1}\right) \cdot n, E_{h k} z_{2}\right)_{E}\right\} d t \\
& =\sum_{n=1}^{N} \int_{I_{n}} \sum_{K \in \overline{\mathcal{T}}_{h}^{n}}\left\{\left(r_{d}, E_{h k} z_{2}\right)_{\partial K}+\left(\sigma_{0}\left(U_{1}\right) \cdot n, E_{h k} z_{2}\right)_{\partial K \cap \Gamma_{\mathrm{N}}}\right\} d t,
\end{array}
$$

where $\mathcal{E}_{I}^{n}, \mathcal{E}_{\Gamma_{\mathrm{N}}}^{n}$ are, respectively, the sets of the interior edges and the edges on the Neumann boundary, corresponding to the triangulation $\overline{\mathcal{T}}_{h}^{n}$. 
For the convolution term in (4.9), similar to (4.10), we have

$$
\begin{aligned}
& \int_{0}^{T} \int_{0}^{t} \mathcal{K}(t-s) a\left(U_{1}(s), E_{h k} z_{2}(t)\right) d s d t \\
&=\sum_{n=1}^{N} \int_{I_{n}} \sum_{j=1}^{n} \int_{t_{j-1}}^{t_{j} \wedge t} \mathcal{K}(t-s) \sum_{K \in \overline{\mathcal{T}}_{h}^{j}} a\left(U_{1}(s), E_{h k} z_{2}(t)\right)_{K} d s d t \\
&=\sum_{n=1}^{N} \int_{I_{n}} \sum_{j=1}^{n} \int_{t_{j-1}}^{t_{j} \wedge t} \mathcal{K}(t-s)\left\{\sum_{E \in \mathcal{E}_{I}^{j}}\left(-\left[\sigma_{0}\left(U_{1}\right) \cdot n\right], E_{h k} z_{2}(t)\right)_{E}\right. \\
&\left.+\sum_{E \in \mathcal{E}_{\Gamma_{\mathrm{N}}}^{j}}\left(\sigma_{0}\left(U_{1}\right) \cdot n, E_{h k} z_{2}(t)\right)_{E}\right\} d s \\
&=\sum_{n=1}^{N} \int_{I_{n}} \sum_{j=1}^{n} \int_{t_{j-1}}^{t_{j} \wedge t} \mathcal{K}(t-s) \sum_{K \in \overline{\mathcal{T}}_{h}^{j}}\left\{\left(r_{d}(s), E_{h k} z_{2}(t)\right)_{\partial K}\right. \\
&+\left.\left(\sigma_{0}\left(U_{1}(s)\right) \cdot n, E_{h k} z_{2}(t)\right)_{\partial K \cap \Gamma_{\mathrm{N}}}\right\} d s d t,
\end{aligned}
$$

that implies

$$
\begin{aligned}
\int_{0}^{T} \int_{0}^{t} \mathcal{K}( & t-s) a\left(U_{1}(s), E_{h k} z_{2}(t)\right) d s d t \\
= & \sum_{n=1}^{N} \int_{I_{n}} \sum_{j=1}^{n} \sum_{K \in \overline{\mathcal{T}}_{h}^{j}}\left\{\left(\int_{t_{j-1}}^{t_{j} \wedge t} \mathcal{K}(t-s) r_{d}(s) d s, E_{h k} z_{2}(t)\right)_{\partial K}\right. \\
& \left.+\left(\int_{t_{j-1}}^{t_{j} \wedge t} \mathcal{K}(t-s) \sigma_{0}\left(U_{1}(s)\right) \cdot n d s, E_{h k} z_{2}(t)\right)_{\partial K \cap \Gamma_{\mathrm{N}}}\right\} d t \\
= & \sum_{n=1}^{N} \sum_{j=1}^{n} \sum_{K \in \overline{\mathcal{T}}_{h}^{j}} \int_{I_{n}}\left(\int_{t_{j-1}}^{t_{j} \wedge t} \mathcal{K}(t-s) r_{d}(s) d s, E_{h k} z_{2}(t)\right)_{\partial K} d t \\
& +\sum_{n=1}^{N} \int_{I_{n}}\left(\int_{0}^{t} \mathcal{K}(t-s) \sigma_{0}\left(U_{1}(s)\right) \cdot n d s, E_{h k} z_{2}(t)\right)_{\Gamma_{\mathrm{N}}} d t \\
= & \sum_{n=1}^{N} \sum_{j=1}^{n} \sum_{K \in \overline{\mathcal{T}}_{h}^{j}} \int_{I_{n}}\left(\int_{t_{j-1}}^{t_{j} \wedge t} \mathcal{K}(t-s) r_{d}(s) d s, E_{h k} z_{2}(t)\right)_{\partial K} d t \\
& +\sum_{n=1}^{N} \int_{I_{n}} \sum_{K \in \overline{\mathcal{T}}_{h}^{n}}\left(\int_{0}^{t} \mathcal{K}(t-s) \sigma_{0}\left(U_{1}(s)\right) \cdot n d s, E_{h k} z_{2}(t)\right)_{\partial K \cap \Gamma_{\mathrm{N}}} d t .
\end{aligned}
$$


This, together with (4.10) and recalling $g_{d}$ from (4.7), imply

$$
\begin{aligned}
\int_{0}^{T}\left\{a\left(U_{1}(s), E_{h k} z_{2}(t)\right)-\int_{0}^{t} \mathcal{K}(t-s) a\left(U_{1}(s), E_{h k} z_{2}(t)\right) d s\right\} d t \\
=\sum_{n=1}^{N} \sum_{K \in \overline{\mathcal{T}}_{h}^{n}}\left\{\left(r_{d}, E_{h k} z_{2}\right)_{\partial K^{n}}+\left(g_{d}, E_{h k} z_{2}\right)_{\partial K^{n}}\right\} \\
\quad-\sum_{n=1}^{N} \sum_{j=1}^{n} \sum_{K \in \overline{\mathcal{T}}_{h}^{j}} \int_{I_{n}}\left(\int_{t_{j-1}}^{t_{j} \wedge t} \mathcal{K}(t-s) r_{d}(s) d s, E_{h k} z_{2}\right)_{\partial K} d t .
\end{aligned}
$$

Hence, from (4.11) and space-time cellwise representation of the other terms in (4.9), we conclude the first a posteriori error representation (4.2).

Now we prove the second error representation (4.3). To this end, in (4.8) we use (2.5) the second variant of the bilinear form $B$, in which the convolution term has been rearanged. Therefore we just need to study the rearanged convolution term that, similar to (4.10), is writen as

$$
\begin{aligned}
\int_{0}^{T} \int_{t}^{T} \mathcal{K} & (s-t) a\left(U_{1}(t), E_{h k} z_{2}(s)\right) d s d t \\
= & \sum_{n=1}^{N} \int_{I_{n}} \sum_{K \in \overline{\mathcal{T}}_{h}^{n}} a\left(U_{1}(t), \int_{t}^{T} \mathcal{K}(s-t) E_{h k} z_{2}(s) d s\right)_{K} d t \\
= & \sum_{n=1}^{N} \int_{I_{n}} \sum_{K \in \overline{\mathcal{T}}_{h}^{n}}\left(r_{d}, \int_{t}^{T} \mathcal{K}(s-t) E_{h k} z_{2}(s) d s\right)_{\partial K} d t \\
& +\sum_{n=1}^{N} \int_{I_{n}}\left(\sigma_{0}\left(U_{1}\right) \cdot n, \int_{t}^{T} \mathcal{K}(s-t) E_{h k} z_{2}(s) d s\right)_{\Gamma_{\mathrm{N}}} d t .
\end{aligned}
$$

For the second term of the right side, we exchange the role of the variables $s, t$, and we change the order of the time integrals to obtain

$$
\begin{aligned}
\sum_{n=1}^{N} \int_{I_{n}} & \left(\sigma_{0}\left(U_{1}\right) \cdot n, \int_{t}^{T} \mathcal{K}(s-t) E_{h k} z_{2}(s) d s\right)_{\Gamma_{\mathrm{N}}} d t \\
= & \int_{0}^{T} \int_{s}^{T} \mathcal{K}(t-s)\left(\sigma_{0}\left(U_{1}(s)\right) \cdot n, E_{h k} z_{2}(t)\right)_{\Gamma_{\mathrm{N}}} d t d s \\
= & \sum_{n=1}^{N} \int_{I_{n}}\left(\int_{0}^{t} \mathcal{K}(t-s) \sigma_{0}\left(U_{1}(s)\right) \cdot n d s, E_{h k} z_{2}(t)\right)_{\Gamma_{\mathrm{N}}} d t
\end{aligned}
$$

that, with (4.12), we have

$$
\begin{aligned}
\int_{0}^{T} \int_{t}^{T} \mathcal{K} & (s-t) a\left(U_{1}(t), E_{h k} z_{2}(s)\right) d s d t \\
= & \sum_{n=1}^{N} \int_{I_{n}} \sum_{K \in \overline{\mathcal{T}}_{h}^{n}}\left\{\left(r_{d}, \int_{t}^{T} \mathcal{K}(s-t) E_{h k} z_{2}(s) d s\right)_{\partial K}\right. \\
& \left.+\left(\int_{0}^{t} \mathcal{K}(t-s) \sigma_{0}\left(U_{1}(s)\right) \cdot n d s, E_{h k} z_{2}(t)\right)_{\partial K \cap \Gamma_{\mathrm{N}}}\right\} d t
\end{aligned}
$$


This, together with (4.10), imply

$$
\begin{gathered}
\int_{0}^{T}\left\{a\left(U_{1}(s), E_{h k} z_{2}(t)\right)-\int_{t}^{T} \mathcal{K}(s-t) a\left(U_{1}(t), E_{h k} z_{2}(s)\right) d s\right\} d t \\
=\sum_{n=1}^{N} \sum_{K \in \overline{\mathcal{T}}_{h}^{n}}\left\{\left(r_{d}, E_{h k} z_{2}\right)_{\partial K^{n}}+\left(g_{d}, E_{h k} z_{2}\right)_{\partial K^{n}}\right. \\
\left.-\left(r_{d}, \int_{t}^{T} \mathcal{K}(s-t) E_{h k} z_{2}(s) d s\right)_{\partial K^{n}}\right\},
\end{gathered}
$$

that is the replacement for (4.11).

Hence, from (4.14) and space-time cellwise representation of the other terms in (4.9), we conclude the second error representation (4.3).

Finally, we prove the third error representation (4.4). In (4.8) we use (2.5), the second variant of the bilinear form $B$, and similar to (4.10) we have

$$
\begin{array}{rl}
\int_{0}^{T} \int_{t}^{T} & \mathcal{K}(s-t) a\left(U_{1}(t), E_{h k} z_{2}(s)\right) d s d t \\
= & \sum_{n=1}^{N} \int_{I_{n}} \sum_{j=n}^{N} \int_{t_{j-1} \vee t}^{t_{j}} \mathcal{K}(s-t) a\left(U_{1}(t), E_{h k} z_{2}(s)\right) d s d t \\
= & \sum_{n=1}^{N} \int_{I_{n}} \sum_{j=n}^{N} \int_{t_{j-1} \vee t}^{t_{j}} \mathcal{K}(s-t) \sum_{K \in \overline{\mathcal{T}}_{h}^{j}}\left\{\left(r_{d}(t), E_{h k} z_{2}(s)\right)_{\partial K}\right. \\
& \left.\quad+\left(\sigma_{0}\left(U_{1}(t)\right) \cdot n, E_{h k} z_{2}(s)\right)_{\partial K \cap \Gamma_{\mathrm{N}}}\right\} d s d t .
\end{array}
$$

Then, rewriting the second term in the brace similar to (4.13), we have

$$
\begin{aligned}
\int_{0}^{T} \int_{t}^{T} \mathcal{K}(s-t) a\left(U_{1}(t), E_{h k} z_{2}(s)\right) d s d t \\
=\sum_{n=1}^{N} \sum_{j=n}^{N} \sum_{K \in \overline{\mathcal{T}}_{h}^{j}} \int_{I_{n}} \int_{t_{j-1} \vee t}^{t_{j}} \mathcal{K}(s-t)\left(r_{d}(t), E_{h k} z_{2}(s)\right)_{\partial K} d s d t \\
\quad+\sum_{n=1}^{N} \int_{I_{n}} \sum_{K \in \overline{\mathcal{T}}_{h}^{n}}\left(\int_{0}^{t} \mathcal{K}(t-s) \sigma_{0}\left(U_{1}(s)\right) \cdot n d s, E_{h k} z_{2}(t)\right)_{\partial K \cap \Gamma_{\mathrm{N}}} d t
\end{aligned}
$$

This, together with 4.10), imply

$$
\begin{aligned}
\int_{0}^{T}\left\{a\left(U_{1}(s), E_{h k} z_{2}(t)\right)-\int_{t}^{T} \mathcal{K}(s-t) a\left(U_{1}(t), E_{h k} z_{2}(s)\right) d s\right\} d t \\
=\sum_{n=1}^{N} \sum_{K \in \overline{\mathcal{T}}_{h}^{n}}\left\{\left(r_{d}, E_{h k} z_{2}\right)_{\partial K^{n}}+\left(g_{d}, E_{h k} z_{2}\right)_{\partial K^{n}}\right\} \\
\quad-\sum_{n=1}^{N} \sum_{j=n}^{N} \sum_{K \in \overline{\mathcal{T}}_{h}^{j}} \int_{I_{n}} \int_{t_{j-1} \vee t}^{t_{j}}\left(r_{d}(t), \mathcal{K}(s-t) E_{h k} z_{2}(s)\right)_{\partial K} d s d t
\end{aligned}
$$

that is the replacement for (4.11). 
Hence, from (4.16) and space-time cellwise representation of the other terms in (4.9), we conclude the third error representation (4.4). Now the proof is complete.

We note that all error indicators in (4.5) depend on the unknown dual solution $z$, which is not available. For strategies to evaluate the error indicators, that are based on suitable approximation of the dual solution, we refer to [5] and [6]. When the spatial meshes change from a time level to the next one, evaluation of functions is carried out by means of interpolation/extrapolation. We recall that one hanging node is allowed per edge or face.

We also note that the difference between a posteriori error representations (4.2)(4.4) are the error indicators $\Theta_{5, K}^{n, j}, \Theta_{5, K}^{n}$, and $\Theta_{5, K}^{N, j}$, where we apply the convolution integral either on the residual $r_{d}$ or on the error term $E_{h k} z_{2}$. Obviously we choose the cheaper one, depending on the method we choose for computing or estimating $E_{h k} z_{2}$. It should be noted that the first and the third error representations (4.2) and (4.4) are not space-time cellwise.

\section{A posteriori error estimates based on global projections}

In order to evaluate the a posteriori error representations (4.2)-(4.4), we need information about the continuous dual solution $z$. Such information has to be obtained either through a priori analysis in form of bounds for $z$ in certain Sobolev norms or through computation by solving the dual problem numerically. In this context we provide information through a priori analysis and we leave the investigation on the second case to a later work.

Sometimes the target functional one is interested in is (almost) global. Then, instead of numerically approximating the dual solution, one can get cheaper error indicators by using analytical a priori estimates for the dual solution. We first present a weighted global a posteriori error estimate, using global $L_{2}$-projections $\mathcal{P}_{k}, \mathcal{P}_{h}$ defined in (3.7), and error estimates of $\mathcal{P}_{h}$ in a weighted $L_{2}$-norm.

We recall the weighted global error estimates of the $L_{2}$-projection $\mathcal{P}_{h}$ (3.7), see 7. First we recall some notation. Let $\mathcal{T}$ be a given triangulation with mesh function $h$, and for any simplex $K \in \mathcal{T}, \rho_{K}$ denote the radius of the largest ball contained in the closure of $K$, that is $\bar{K}$. A family $\mathcal{F}$ of triangulations $\mathcal{T}$ is called non-degenerate, if there exsists a constant $c_{0}$ such that

$$
c_{0}=\max _{\mathcal{T} \in \mathcal{F}} \max _{K \in \mathcal{T}} \frac{h_{K}}{\rho_{K}} .
$$

Let $S_{K}=\left\{K^{\prime} \in \mathcal{T}: \bar{K}^{\prime} \cap \bar{K} \neq \varnothing\right\}$. Also $\delta_{\mathcal{T}}$ and $\delta_{\mathcal{F}}$ be measures for a given triangulation $\mathcal{T}$ and a given family $\mathcal{F}$, respectively, defined by

$$
\delta_{\mathcal{T}}=\max _{K \in \mathcal{T}} \max _{K^{\prime} \in S_{K}}\left|1-h_{K^{\prime}}^{2} / h_{K}^{2}\right|, \quad \delta_{\mathcal{F}}=\max _{\mathcal{T} \in \mathcal{F}} \delta_{\mathcal{T}} .
$$

We define the error operators $E_{h k}, E_{h}$, and $E_{k}$ by

$$
E_{h k} v=\left(\mathcal{P}_{k} \mathcal{P}_{h}-I\right) v, \quad E_{h} v=\left(\mathcal{P}_{h}-I\right) v, \quad E_{k} v=\left(\mathcal{P}_{k}-I\right) v,
$$

and we note that

$$
E_{h k}=E_{h}+E_{k} \mathcal{P}_{h} .
$$


We recall standard error estimation for the time projection error $E_{k}$, that is,

$$
\int_{I_{n}}\left|E_{k} v\right| d t=\int_{I_{n}}\left|E_{k, n} v\right| d t \leq C k_{n}^{\gamma} \int_{I_{n}}\left|\partial_{t}^{\gamma} v\right| d t, \quad \gamma=0,1
$$

We also quote the error estimates for the spatial projection error $E_{h}$ from [7], which is stated in the following lemma.

Lemma 2. Assume that the family $\mathcal{F}$ of traingulations $\mathcal{T}$ be non-degenerate. Then for sufficiently small $\delta_{\mathcal{F}}$, there exists a constant $C$ such that for any triangulation $\mathcal{T} \in \mathcal{F}$ we have,

$$
\begin{gathered}
\left\|h^{-s} E_{h} v\right\| \leq C\left\|\nabla^{s} v\right\|, \quad s=0,1,2, \forall v \in H^{s}, \\
\left\|h^{-s} \nabla E_{h} v\right\| \leq C\left\|\nabla^{s+1} v\right\|, \quad s=0,1, \forall v \in H^{s}
\end{gathered}
$$

where ' $\nabla$ ' denotes the usual gradient.

For more details on the practical aspects of $\delta_{\mathcal{F}}$, see 7 .

For the next theorem we recall the mesh functions $h_{n}, \bar{h}_{n}$ from (3.1), (3.2), and we define the notations

$$
\begin{array}{ll}
\bar{h}_{\min , n}=\min _{K \in \overline{\mathcal{T}}_{h}^{n}} \bar{h}_{K}, & \bar{h}_{\max , n}=\max _{K \in \overline{\mathcal{T}}_{h}^{n}} \bar{h}_{K}, \\
\mathcal{K}_{n, T}=\left(\int_{t}^{T} \mathcal{K}(s-t) d s\right)^{1 / 2}, & \mathcal{K}_{n, j}=\left(\int_{t \vee t_{j-1}}^{t_{j}} \mathcal{K}(s-t) d s\right)^{1 / 2} .
\end{array}
$$

We also use the scaled trace inequality, for any simplex $K \in \mathcal{T}_{h}$,

$$
\|v\|_{\partial K} \leq C\left(h_{K}^{-1 / 2}\|v\|_{K}+h_{K}^{1 / 2}\|\nabla v\|_{K}\right)
$$

Theorem 2. Let $u$ be the solution of (2.6), and $U$ be the solution of (3.4) with a non-degenerate family $\mathcal{F}_{h}$ of triangulations $\mathcal{T}_{h}^{n}, n=0,1, \ldots, N$, with sufficiently small $\delta_{\mathcal{F}_{h}}$, such that the weighted global error estimates (5.3) and (5.4) hold. We also assume that $\mathcal{K} \in L_{1}\left(\mathbb{R}^{+}\right)$. Then, denoting the error $e=U-u$, we have the weighted a posteriori error estimate, for $\alpha=0,1,2, \beta=1,2, \gamma=0,1$,

$$
\begin{aligned}
\left|L^{*}(e)\right| \leq & C \max _{0 \leq t \leq T}\left\{\left\|\nabla^{\alpha} z_{1}(t)\right\|,\left\|\nabla^{\beta} z_{2}(t)\right\|,\left\|\partial_{t}^{\alpha \wedge 1} z_{1}(t)\right\|,\left\|\partial_{t}^{\gamma} z_{2}(t)\right\|\right\} \\
& \times\left\{\Upsilon_{0}+\sum_{n=1}^{N} \int_{I_{n}}\left(\Upsilon_{h}+\Upsilon_{h, \partial K}+\Upsilon_{k}+\Upsilon_{k, \partial K}\right) d t\right\}
\end{aligned}
$$


where

$$
\begin{aligned}
& \Upsilon_{0}=\left\|h_{0}^{\alpha}\left(U_{1}(0)-u^{0}\right)\right\|+\left\|h_{0}^{\beta}\left(U_{2}(0)-v^{0}\right)\right\|, \\
& \Upsilon_{h}=\left\|\bar{h}_{n}^{\alpha}\left(\dot{U}_{1}-U_{2}\right)\right\|+\left\|\bar{h}_{n}^{\beta}\left(\dot{U}_{2}-f\right)\right\|, \\
& \Upsilon_{h, \partial K}=\left(\zeta_{n}(\beta)+\zeta_{n, N}(\beta)\right)\left(\sum_{K \in \overline{\mathcal{T}}_{h}^{n}} \bar{h}_{K}^{3}\left\|r_{d}\right\|_{\partial K}^{2}\right)^{1 / 2} \\
& \quad+\zeta_{n}(\beta)\left(\sum_{K \in \overline{\mathcal{T}}_{h}^{n}} \bar{h}_{K}^{3}\left\|g_{d}-g\right\|_{\partial K}^{2}\right)^{1 / 2}, \\
& \Upsilon_{k}=k_{n}^{\alpha \wedge 1}\left\|E_{k}\left(\dot{U}_{1}-U_{2}\right)\right\|+k_{n}^{\gamma}\left(\left\|E_{k} \bar{A}_{h} U_{1}\right\|\right. \\
& \left.\quad+\left\|E_{k} \int_{0}^{t} \mathcal{K}(t-s) \bar{A}_{h} U_{1}(s) d s\right\|+\left\|E_{k} f\right\|\right), \\
& \Upsilon_{k, \partial K}=k_{n}^{\gamma}\left(\sum_{K \in \overline{\mathcal{T}}_{h}^{n}} h_{K}^{-1}\left\|E_{k} g\right\|_{\partial K}^{2}\right)^{1 / 2},
\end{aligned}
$$

with $\zeta_{n}(\beta)=\bar{h}_{\min , n}^{\beta-2}, \zeta_{n, N}(\beta)=\bar{h}_{\min , n}^{-3 / 2} \mathcal{K}_{n, T} \sum_{j=n}^{N} \mathcal{K}_{n, j} \bar{h}_{\max , j}^{\beta-\frac{1}{2}}$.

Proof. Let $z \in \mathcal{V}^{*}$ be the solution of the dual problem (2.10). From the definition of the $L_{2}$ projections $\mathcal{P}_{k}, \mathcal{P}_{h}$ in (3.7) and the test space $\mathcal{W}_{h k}$ in (3.3) we have $\mathcal{P}_{k} \mathcal{P}_{h} z \in \mathcal{W}_{h k}$. Therefore, using (4.8) and (5.1) we have,

$$
L^{*}(e)=R\left(U ; E_{h k} z\right)=R\left(U ; E_{h} z\right)+R\left(U ; E_{k} \mathcal{P}_{h} z\right)
$$

We study the two terms at the right side of this equation.

For the first term, recalling (4.8) and using the a posteriori error representation (4.3), we have

$$
\begin{aligned}
\mathrm{R}\left(U ; E_{h} z\right)= & L^{*}(e) \\
= & \sum_{K \in \mathcal{T}_{h}^{0}}\left\{\left(U_{1}(0)-u^{0}, E_{h} z_{1}(0)\right)_{K}+\left(U_{2}(0)-v^{0}, E_{h} z_{2}(0)\right)_{K}\right\} \\
& +\sum_{n=1}^{N} \int_{I_{n}} \sum_{K \in \overline{\mathcal{T}}_{h}^{n}}\left\{\left(\dot{U}_{1}-U_{2}, E_{h} z_{1}\right)_{K}+\left(\dot{U}_{2}-f, E_{h} z_{2}\right)_{K}\right. \\
& +\left(r_{d}, E_{h} z_{2}\right)_{\partial K}+\left(g_{d}-g, E_{h} z_{2}\right)_{\partial K} \\
& \left.\quad-\left(r_{d}, \int_{t}^{T} \mathcal{K}(s-t) E_{h} z_{2}(s) d s\right)_{\partial K}\right\} d t=\sum_{i=1}^{2} \mathbf{I}_{i}+\sum_{i=1}^{5} \mathbf{I I}_{i} .
\end{aligned}
$$

We then, for each term, use the Cauchy-Schwarz inequality twice. First on the local elements $K$ and $\partial K$, to obtain local $L_{2}$-norms, and then on the sum over the elements to obtain global norms such that the weighted global error estimates (5.3) - (5.4) can be used. For $\mathbf{I}_{1}$, using (5.3), we have, for $\alpha=0,1,2$,

$$
\mathbf{I}_{1} \leq\left\|h_{0}^{\alpha}\left(U_{1}(0)-u^{0}\right)\right\|\left\|h_{0}^{-\alpha} E_{h} z_{1}(0)\right\| \leq C\left\|h_{0}^{\alpha}\left(U_{1}(0)-u^{0}\right)\right\|\left\|\nabla^{\alpha} z_{1}(0)\right\|,
$$

and in a similar way, for $\beta=1,2$,

$$
\mathbf{I}_{2} \leq C\left\|h_{0}^{\beta}\left(U_{2}(0)-v^{0}\right)\right\|\left\|\nabla^{\beta} z_{2}(0)\right\| .
$$


Now we estimate the second terms $\mathbf{I I}_{i}, i=1, \ldots, 5$. Using the Cauchy-Schwarz inequality and the error estimate (5.3), we have, for $\alpha=0,1,2$,

$$
\begin{aligned}
\mathbf{I I}_{1} & =\sum_{n=1}^{N} \int_{I_{n}} \sum_{K \in \overline{\mathcal{T}}_{h}^{n}}\left(\dot{U}_{1}-U_{2}, E_{h} z_{1}\right)_{K} d t \\
& \leq \sum_{n=1}^{N} \int_{I_{n}}\left\|\bar{h}_{n}^{\alpha}\left(\dot{U}_{1}-U_{2}\right)\right\|\left\|\bar{h}_{n}^{-\alpha} E_{h} z_{1}\right\| d t \\
& \leq C \max _{[0, T]}\left\|\nabla^{\alpha} z_{1}(t)\right\| \sum_{n=1}^{N} \int_{I_{n}}\left\|\bar{h}_{n}^{\alpha}\left(\dot{U}_{1}-U_{2}\right)\right\| d t,
\end{aligned}
$$

and similarly we obtain, for $\beta=1,2$,

$$
\mathbf{I I}_{2} \leq C \max _{[0, T]}\left\|\nabla^{\beta} z_{2}(t)\right\| \sum_{n=1}^{N} \int_{I_{n}}\left\|\bar{h}_{n}^{\beta}\left(\dot{U}_{2}-f\right)\right\| d t .
$$

For $\mathbf{I I}_{3}$, we first have,

$$
\mathbf{I I}_{3} \leq \sum_{n=1}^{N} \int_{I_{n}}\left(\sum_{K \in \overline{\mathcal{T}}_{h}^{n}} \bar{h}_{K}^{3}\left\|r_{d}(t)\right\|_{\partial K}^{2}\right)^{1 / 2}\left(\sum_{K \in \overline{\mathcal{T}}_{h}^{n}} \bar{h}_{K}^{-3}\left\|E_{h} z_{2}(t)\right\|_{\partial K}^{2}\right)^{1 / 2} d t .
$$

Then, by the scaled trace inequality (5.5) and the weighted global error estimates (5.3)-(5.4), we obtain, for $\beta=1,2$,

$$
\begin{aligned}
\sum_{K \in \overline{\mathcal{T}}_{h}^{n}} \bar{h}_{K}^{-3}\left\|E_{h} z_{2}\right\|_{\partial K}^{2} & \leq C \sum_{K \in \overline{\mathcal{T}}_{h}^{n}}\left\{\bar{h}_{K}^{-4}\left\|E_{h} z_{2}\right\|_{K}^{2}+\bar{h}_{K}^{-2}\left\|\nabla E_{h} z_{2}\right\|_{K}^{2}\right\} \\
& \leq C \sum_{K \in \overline{\mathcal{T}}_{h}^{n}} \bar{h}_{K}^{-4+2 \beta}\left\{\left\|\bar{h}_{K}^{-\beta} E_{h} z_{2}\right\|_{K}^{2}+\left\|\bar{h}_{K}^{-\beta+1} \nabla E_{h} z_{2}\right\|_{K}^{2}\right\} \\
& \leq C \bar{h}_{m i n, n}^{2(\beta-2)}\left\|\nabla^{\beta} z_{2}\right\|^{2} .
\end{aligned}
$$

These imply the estimate, for $\beta=1,2$,

$$
\mathbf{I I}_{3} \leq C \max _{[0, T]}\left\|\nabla^{\beta} z_{2}(t)\right\| \sum_{n=1}^{N} \int_{I_{n}} \bar{h}_{\min , n}^{\beta-2}\left(\sum_{K \in \overline{\mathcal{T}}_{h}^{n}} \bar{h}_{K}^{3}\left\|r_{d}\right\|_{\partial K}^{2}\right)^{1 / 2} d t .
$$

In a similar way, we have, for $\beta=1,2$,

$$
\mathbf{I I}_{4} \leq C \max _{[0, T]}\left\|\nabla^{\beta} z_{2}(t)\right\| \sum_{n=1}^{N} \int_{I_{n}} \bar{h}_{\min , n}^{\beta-2}\left(\sum_{K \in \overline{\mathcal{T}}_{h}^{n}} \bar{h}_{K}^{3}\left\|g_{d}-g\right\|_{\partial K}^{2}\right)^{1 / 2} d t .
$$

Finally we study $\mathbf{I I}_{5}$. To this end, first we note that,

$$
\begin{aligned}
\mathbf{I I}_{5} \leq & \sum_{n=1}^{N} \int_{I_{n}}\left(\sum_{K \in \overline{\mathcal{T}}_{h}^{n}} \bar{h}_{K}^{3}\left\|r_{d}(t)\right\|_{\partial K}^{2}\right)^{1 / 2} \\
& \times\left(\sum_{K \in \overline{\mathcal{T}}_{h}^{n}} \bar{h}_{K}^{-3}\left\|\int_{t}^{T} \mathcal{K}(s-t) E_{h} z_{2}(s) d s\right\|_{\partial K}^{2}\right)^{1 / 2} d t .
\end{aligned}
$$


Then, using the Cuachy-Schwarz inequality, we have

$$
\begin{aligned}
\sum_{K \in \overline{\mathcal{T}}_{h}^{n}} \bar{h}_{K}^{-3}\left\|\int_{t}^{T} \mathcal{K}(s-t) E_{h} z_{2}(s) d s\right\|_{\partial K}^{2} \\
\leq \sum_{K \in \overline{\mathcal{T}}_{h}^{n}} \bar{h}_{K}^{-3}\left(\int_{t}^{T} \mathcal{K}(s-t)\left\|E_{h} z_{2}(s)\right\|_{\partial K} d s\right)^{2} \\
\leq \sum_{K \in \overline{\mathcal{T}}_{h}^{n}} \bar{h}_{K}^{-3} \int_{t}^{T} \mathcal{K}(s-t) d s \int_{t}^{T} \mathcal{K}(s-t)\left\|E_{h} z_{2}(s)\right\|_{\partial K}^{2} d s \\
\leq \mathcal{K}_{n, T}^{2} \int_{t}^{T} \mathcal{K}(s-t) \sum_{K \in \overline{\mathcal{T}}_{h}^{n}} \bar{h}_{K}^{-3}\left\|E_{h} z_{2}(s)\right\|_{\partial K}^{2} d s,
\end{aligned}
$$

that using the scaled trace inequality (5.5) and (5.3) -(5.4), we have, for $\beta=1,2$,

$$
\begin{aligned}
& \sum_{K \in \overline{\mathcal{T}}_{h}^{n}} \bar{h}_{K}^{-3}\left\|\int_{t}^{T} \mathcal{K}(s-t) E_{h} z_{2}(s) d s\right\|_{\partial K}^{2} \\
& \leq C \bar{h}_{m i n, n}^{-3} \mathcal{K}_{n, T}^{2} \sum_{j=n}^{N} \int_{t \vee t_{j-1}}^{t_{j}} \mathcal{K}(s-t) \sum_{K \in \overline{\mathcal{T}}_{h}^{j}}\left\{\bar{h}_{K}^{-1}\left\|E_{h} z_{2}(s)\right\|_{K}^{2}\right. \\
& \left.+\bar{h}_{K}\left\|\nabla E_{h} z_{2}(s)\right\|_{K}^{2}\right\} d s \\
& \leq C \bar{h}_{m i n, n}^{-3} \mathcal{K}_{n, T}^{2} \sum_{j=n}^{N} \int_{t \vee t_{j-1}}^{t_{j}} \mathcal{K}(s-t) \sum_{K \in \overline{\mathcal{T}}_{h}^{j}} \bar{h}_{K}^{-1+2 \beta}\left\{\left\|\bar{h}_{K}^{-\beta} E_{h} z_{2}(s)\right\|_{K}^{2}\right. \\
& \left.+\left\|\bar{h}_{K}^{-\beta+1} \nabla E_{h} z_{2}(s)\right\|_{K}^{2}\right\} d s \\
& \leq C \bar{h}_{\text {min }, n}^{-3} \mathcal{K}_{n, T}^{2} \sum_{j=n}^{N} \bar{h}_{\max , j}^{2\left(\beta-\frac{1}{2}\right)} \int_{t \vee t_{j-1}}^{t_{j}} \mathcal{K}(s-t)\left\|\nabla^{\beta} z_{2}(s)\right\|^{2} d s \\
& \leq C \bar{h}_{\min , n}^{-3} \mathcal{K}_{n, T}^{2} \max _{[t, T]}\left\|\nabla^{\beta} z_{2}(s)\right\|^{2} \sum_{j=n}^{N} \bar{h}_{\max , j}^{2\left(\beta-\frac{1}{2}\right)} \int_{t \vee t_{j-1}}^{t_{j}} \mathcal{K}(s-t) d s .
\end{aligned}
$$

Hence we have

$$
\begin{aligned}
\mathbf{I I}_{5} \leq & C \max _{[0, T]}\left\|\nabla^{\beta} z_{2}(t)\right\| \\
& \times \sum_{n=1}^{N} \int_{I_{n}}\left\{\bar{h}_{\min , n}^{-3 / 2} \mathcal{K}_{n, T} \sum_{j=n}^{N}\left(\bar{h}_{\max , j}^{\beta-\frac{1}{2}} \mathcal{K}_{n, j}\right)\left(\sum_{K \in \overline{\mathcal{T}}_{h}^{n}} \bar{h}_{K}^{3}\left\|r_{d}\right\|_{\partial K}^{2}\right)^{1 / 2}\right\} d t .
\end{aligned}
$$


Putting (5.9) -(5.15) in (5.8) we conclude, for $\alpha=0,1,2, \beta=1,2$,

$$
\begin{aligned}
& \mathrm{R}\left(U ; E_{h} z\right) \leq C \max _{[0, T]}\left\{\left\|\nabla^{\alpha} z_{1}(t)\right\|,\left\|\nabla^{\beta} z_{2}(t)\right\|\right\} \\
& \times\left\{\left\|h_{0}^{\alpha}\left(U_{1}(0)-u^{0}\right)\right\|+\left\|h_{0}^{\beta}\left(U_{2}(0)-v^{0}\right)\right\|\right. \\
& \quad+\sum_{n=1}^{N} \int_{I_{n}}\left\{\left\|\bar{h}_{n}^{\alpha}\left(\dot{U}_{1}-U_{2}\right)\right\|+\left\|\bar{h}_{n}^{\beta}\left(\dot{U}_{2}-f\right)\right\|\right. \\
& \quad+\bar{h}_{\min , n}^{\beta-2}\left(\sum_{K \in \overline{\mathcal{T}}_{h}^{n}} \bar{h}_{K}^{3}\left\|r_{d}\right\|_{\partial K}^{2}\right)^{1 / 2}+\bar{h}_{\min , n}^{\beta-2}\left(\sum_{K \in \overline{\mathcal{T}}_{h}^{n}} \bar{h}_{K}^{3}\left\|g_{d}-g\right\|_{\partial K}^{2}\right)^{1 / 2} \\
& \left.\left.\quad+\bar{h}_{\min , n}^{-3 / 2} \mathcal{K}_{n, T} \sum_{j=n}^{N}\left(\bar{h}_{\max , j}^{\beta-\frac{1}{2}} \mathcal{K}_{n, j}\right)\left(\sum_{K \in \overline{\mathcal{T}}_{h}^{n}} \bar{h}_{K}^{3}\left\|r_{d}\right\|_{\partial K}^{2}\right)^{1 / 2}\right\} d t\right\} .
\end{aligned}
$$

Now we study the second term $R\left(U ; E_{k} P_{h} z\right)$ in (5.7). We have

$$
\begin{aligned}
\mathrm{R}\left(U ; E_{k} \mathcal{P}_{h} z\right)= & \mathrm{R}\left(U ; E_{k} \mathcal{P}_{h} z\right) \pm \int_{0}^{T}\left\{\left(\mathcal{P}_{k} f, E_{k} \mathcal{P}_{h} z_{2}\right)+\left(\mathcal{P}_{k} g, E_{k} \mathcal{P}_{h} z_{2}\right)_{\Gamma_{\mathrm{N}}}\right\} d t \\
= & \left(U_{1}(0)-u^{0}, E_{k} \mathcal{P}_{h} z_{1}(0)\right)+\left(U_{2}(0)-v^{0}, E_{k} \mathcal{P}_{h} z_{2}(0)\right) \\
& +\sum_{n=1}^{N} \int_{I_{n}}\left\{\left(\dot{U}_{1}-U_{2}, E_{k} \mathcal{P}_{h} z_{1}\right)+a\left(U_{1}, E_{k} \mathcal{P}_{h} z_{2}\right)\right. \\
& -\int_{0}^{t} \mathcal{K}(t-s) a\left(U_{1}(s), E_{k} \mathcal{P}_{h} z_{2}\right) d s+\left(\dot{U}_{2}-\mathcal{P}_{k} f, E_{k} \mathcal{P}_{h} z_{2}\right) \\
& \left.-\left(\mathcal{P}_{k} g, E_{k} \mathcal{P}_{h} z_{2}\right)_{\Gamma_{\mathrm{N}}}+\left(E_{k} f, E_{k} \mathcal{P}_{h} z_{2}\right)+\left(E_{k} g, E_{k} \mathcal{P}_{h} z_{2}\right)_{\Gamma_{\mathrm{N}}}\right\} d t .
\end{aligned}
$$

Recalling the initial data (3.8), $U_{i}(0)=\mathcal{P}_{h} u_{i}(0), i=1,2$, the first two terms on the right side vanish. Besides, from the second equation of (3.6) we have, for $V \in V_{h}^{n}$,

$$
\begin{aligned}
\int_{I_{n}}\left\{\left(\dot{U}_{2}, V\right)-\left(\mathcal{P}_{k} f, V\right)-\left(\mathcal{P}_{k} g, V\right)_{\Gamma_{\mathrm{N}}}\right\} d t \\
\quad=-\int_{I_{n}}\left\{a\left(\mathcal{P}_{k} U_{1}, V\right)-a\left(\mathcal{P}_{k} \int_{0}^{t} \mathcal{K}(t-s) U_{1}(s) d s, V\right)\right\} d t .
\end{aligned}
$$

Hence, we conclude

$$
\begin{aligned}
\mathrm{R}\left(U ; E_{k} \mathcal{P}_{h} z\right)=\sum_{n=1}^{N} & \int_{I_{n}}\left\{\left(E_{k}\left(\dot{U}_{1}-U_{2}\right), E_{k} \mathcal{P}_{h} z_{1}\right)-a\left(E_{k} U_{1}, E_{k} \mathcal{P}_{h} z_{2}\right)\right. \\
& +a\left(E_{k} \int_{0}^{t} \mathcal{K}(t-s) U_{1}(s) d s, E_{k} \mathcal{P}_{h} z_{2}\right) \\
& \left.+\left(E_{k} f, E_{k} \mathcal{P}_{h} z_{2}\right)+\left(E_{k} g, E_{k} \mathcal{P}_{h} z_{2}\right)_{\Gamma_{\mathrm{N}}}\right\} d t
\end{aligned}
$$


For the last term, we have

$$
\begin{aligned}
& \sum_{n=1}^{N} \int_{I_{n}}\left(E_{k} g, E_{k} \mathcal{P}_{h} z_{2}\right)_{\Gamma_{\mathrm{N}}} d t \\
& \quad \leq \sum_{n=1}^{N} \int_{I_{n}}\left(\sum_{K \in \overline{\mathcal{T}}_{h}^{n}} \bar{h}_{K}^{-1}\left\|E_{k} g\right\|_{\partial K}^{2}\right)^{1 / 2}\left(\sum_{K \in \overline{\mathcal{T}}_{h}^{n}} \bar{h}_{K}\left\|E_{k} \mathcal{P}_{h} z_{2}\right\|_{\partial K \cap \Gamma_{\mathrm{N}}}^{2}\right)^{1 / 2} .
\end{aligned}
$$

By the scaled trace inequality (5.5) and local inverse inequality,

$$
h_{K}\|\nabla \varphi\|_{K} \leq C\|\varphi\|_{K}, \quad \forall K \in \overline{\mathcal{T}}_{h}^{n}, \forall \varphi \in \bar{V}_{h}^{n},
$$

we have

$$
\begin{aligned}
\sum_{K \in \overline{\mathcal{T}}_{h}^{n}} \bar{h}_{K}\left\|E_{k} \mathcal{P}_{h} z_{2}\right\|_{\partial K \cap \Gamma_{\mathrm{N}}}^{2} & \leq C \sum_{K \in \overline{\mathcal{T}}_{h}^{n}}\left\{\left\|E_{k} \mathcal{P}_{h} z_{2}\right\|_{K}^{2}+h_{K}^{2}\left\|\nabla E_{k} \mathcal{P}_{h} z_{2}\right\|_{K}^{2}\right\} \\
& \leq C \sum_{K \in \overline{\mathcal{T}}_{h}^{n}}\left\{\left\|E_{k} \mathcal{P}_{h} z_{2}\right\|_{K}^{2}+\left\|E_{k} \mathcal{P}_{h} z_{2}\right\|_{K}^{2}\right\}=C\left\|E_{k} \mathcal{P}_{h} z_{2}\right\|^{2}
\end{aligned}
$$

Hence

$$
\sum_{n=1}^{N} \int_{I_{n}}\left(E_{k} g, E_{k} \mathcal{P}_{h} z_{2}\right)_{\Gamma_{\mathrm{N}}} d t \leq C \sum_{n=1}^{N} \int_{I_{n}}\left(\sum_{K \in \overline{\mathcal{T}}_{h}^{n}} \bar{h}_{K}^{-1}\left\|E_{k} g\right\|_{\partial K}^{2}\right)^{1 / 2}\left\|E_{k} \mathcal{P}_{h} z_{2}\right\| .
$$

Considering this in (5.17), using the Cauchy-Schwarz inequality we have

$$
\begin{aligned}
\mathrm{R}\left(U ; E_{k} \mathcal{P}_{h} z\right) \leq & C \sum_{n=1}^{N} \int_{I_{n}}\left\{\left\|E_{k}\left(\dot{U}_{1}-U_{2}\right)\right\|\left\|E_{k} \mathcal{P}_{h} z_{1}\right\|+\left\|E_{k} \bar{A}_{h} U_{1}\right\|\left\|E_{k} \mathcal{P}_{h} z_{2}\right\|\right. \\
& +\left\|E_{k} \int_{0}^{t} \mathcal{K}(t-s) \bar{A}_{h} U_{1}(s) d s\right\|\left\|E_{k} \mathcal{P}_{h} z_{2}\right\|+\left\|E_{k} f\right\|\left\|E_{k} \mathcal{P}_{h} z_{2}\right\| \\
& \left.+\left(\sum_{K \in \overline{\mathcal{T}}_{h}^{n}} h_{K}^{-1}\left\|E_{k} g\right\|_{\partial K}^{2}\right)^{1 / 2}\left\|E_{k} \mathcal{P}_{h} z_{2}\right\|\right\} .
\end{aligned}
$$

From this, together with $L_{2}$-stability of the $L_{2}$-projection $\mathcal{P}_{h}$ and the error estimate (5.2) for $E_{k}=\mathcal{P}_{k}-I$, we conclude, for $\alpha=0,1,2, \gamma=0,1$,

$$
\begin{aligned}
\mathrm{R}\left(U ; E_{k} \mathcal{P}_{h} z\right) \leq C & \max _{[0, T]}\left\{\left\|\partial_{t}^{\alpha \wedge 1} z_{1}(t)\right\|,\left\|\partial_{t}^{\gamma} z_{2}(t)\right\|\right\} \sum_{n=1}^{N} \int_{I_{n}}\left\{k_{n}^{\alpha \wedge 1}\left\|\dot{U}_{1}-U_{2}\right\|\right. \\
+ & k_{n}^{\gamma}\left\|E_{k} \bar{A}_{h} U_{1}\right\|+k_{n}^{\gamma}\left\|E_{k} \int_{0}^{t} \mathcal{K}(t-s) \bar{A}_{h} U_{1}(s) d s\right\| \\
& \left.+k_{n}^{\gamma}\left\|E_{k} f\right\|+k_{n}^{\gamma}\left(\sum_{K \in \overline{\mathcal{T}}_{h}^{n}} h_{K}^{-1}\left\|E_{k} g\right\|_{\partial K}^{2}\right)^{1 / 2}\right\} d t .
\end{aligned}
$$

Hence, putting (5.16) and (5.18) in (5.7), we conclude the a posteriori error estimate (5.6), and this completes the proof.

We note that, to obtaining a computable error bound in (5.6), one aspect is to estimate or eliminate the norms of the derivative of the dual solutions $z_{1}, z_{2}$. In practice, exact solutions $z_{1}, z_{2}$ are not available. Therefore one way is to use accurate numerical approximations for $z_{1}, z_{2}$, and further the derivatives can be approximated by corresponding difference quotients, see [5] and references therein 
for practical methods for hyperbolic equations. Another possible way is to eliminate these terms by means of stability estimates.

Example 1. For example, let assume that the desired output functional $L^{*}(e)$ is $\left\|e_{1}(T)\right\|$. One way, see [5] for other alternatives, is to set $j_{1}=j_{2}=z_{2}^{T}=0$ and $z_{1}^{T}=e_{1}(T)$ in (5.6). Then, with $\alpha=0, \beta=\gamma=1$, and using the stability estimate (2.14) with $l=0$, we obtain the a posteriori error estimate

$$
\begin{aligned}
\left\|e_{1}(T)\right\| \leq & C\left\{\left\|U_{1}(0)-u^{0}\right\|+\left\|h_{0}\left(U_{2}(0)-v^{0}\right)\right\|\right. \\
+ & \sum_{n=1}^{N} \int_{I_{n}}\left\{\left\|\dot{U}_{1}-U_{2}\right\|+\left\|\bar{h}_{n}\left(\dot{U}_{2}-f\right)\right\|+\zeta_{n}(1)\left(\sum_{K \in \overline{\mathcal{T}}_{h}^{n}} \bar{h}_{K}^{3}\left\|g_{d}-g\right\|_{\partial K}^{2}\right)^{1 / 2}\right. \\
& +\left(\zeta_{n}(1)+\zeta_{n, N}(1)\right)\left(\sum_{K \in \overline{\mathcal{T}}_{h}^{n}} \bar{h}_{K}^{3}\left\|r_{d}\right\|_{\partial K}^{2}\right)^{1 / 2} \\
& +\left\|E_{k}\left(\dot{U}_{1}-U_{2}\right)\right\|+k_{n}\left\|E_{k} \bar{A}_{h} U_{1}\right\|+k_{n}\left\|E_{k} \int_{0}^{t} \mathcal{K}(t-s) \bar{A}_{h} U_{1}(s) d s\right\| \\
& \left.\left.+k_{n}\left\|E_{k} f\right\|+k_{n}\left(\sum_{K \in \overline{\mathcal{T}}_{h}^{n}} h_{K}^{-1}\left\|E_{k} g\right\|_{\partial K}^{2}\right)^{1 / 2}\right\} d t\right\} .
\end{aligned}
$$

Remark 1. We note that when the convolution kenel is slightly more regular such that $\|\mathcal{K}\|_{L_{2}\left(\mathbb{R}^{+}\right)}<\infty$, following the proof of (5.15), we can replace $\zeta_{n, N}(\beta)$ in (5.6) by

$$
\zeta_{n, N}(\beta)=\bar{h}_{\min , n}^{-3 / 2}\left(\int_{t}^{T} \mathcal{K}^{2}(s-t) d s\right)^{1 / 2} \sum_{j=n}^{N} k_{j}^{\frac{1}{2}} \bar{h}_{\max , j}^{\beta-\frac{1}{2}}
$$

Remark 2. In the a posteriori estimate (5.6), with $\beta=2$, we have $\zeta_{n}(2)=1$, but still $\zeta_{n, N}(2)$ can be big when the spatial meshes change. We recall that the error representaion (4.3) has been used for (5.8). If we use the error representaion (4.2), instead, the error indicator

$$
\zeta_{n, N}(\beta)\left(\sum_{K \in \overline{\mathcal{T}}_{h}^{n}} \bar{h}_{K}^{3}\left\|r_{d}\right\|_{\partial K}^{2}\right)^{1 / 2}
$$

using the notation (4.1), is replaced by

$$
\sum_{j=1}^{n} \zeta_{j}(\beta)\left(\sum_{K \in \overline{\mathcal{T}}_{h}^{j}} \bar{h}_{K}^{3}\left\|\left(\mathcal{K} * r_{d}\right)^{j}(t)\right\|_{\partial K}^{2}\right)^{1 / 2}
$$


Indeed for $\mathbf{I I}_{5}$, using the Cauchy-Schwarz inequality and (5.3), we can obtain

$$
\begin{aligned}
\mathbf{I I}_{5} & =\sum_{n=1}^{N} \sum_{j=1}^{n} \sum_{K \in \overline{\mathcal{T}}_{h}^{j}} \int_{I_{n}}\left(\int_{t_{j-1}}^{t_{j} \wedge t} \mathcal{K}(t-s) r_{d}(s) d s, E_{h} z_{2}\right)_{\partial K} d t \\
& \leq \sum_{n=1}^{N} \int_{I_{n}} \sum_{j=1}^{n} \sum_{K \in \overline{\mathcal{T}}_{h}^{j}}\left\|\int_{t_{j-1}}^{t_{j} \wedge t} \mathcal{K}(t-s) r_{d}(s) d s\right\|_{\partial K}\left\|E_{h} z_{2}\right\|_{\partial K} d t \\
& \leq \sum_{n=1}^{N} \int_{I_{n}} \sum_{j=1}^{n}\left(\sum_{K \in \overline{\mathcal{T}}_{h}^{j}} \bar{h}_{K}^{3}\left\|\left(\mathcal{K} * r_{d}\right)^{j}(t)\right\|_{\partial K}^{2}\right)^{1 / 2}\left(\sum_{K \in \overline{\mathcal{T}}_{h}^{j}} \bar{h}_{K}^{-3}\left\|E_{h} z_{2}\right\|_{\partial K}^{2}\right)^{1 / 2} d t \\
& \leq C \max _{[0, T]}\left\|\nabla^{\beta} z_{2}(t)\right\| \sum_{n=1}^{N} \int_{I_{n}} \sum_{j=1}^{n} \bar{h}_{m i n, j}^{\beta-2}\left(\sum_{K \in \overline{\mathcal{T}}_{h}^{j}} \bar{h}_{K}^{3}\left\|\left(\mathcal{K} * r_{d}\right)^{j}(t)\right\|_{\partial K}^{2}\right)^{1 / 2} d t .
\end{aligned}
$$

Hence with $\beta=2$ we get the optimal order error indicators, though this error indicator is not space-time cellwise.

Remark 3. We note that for the error estimate (5.6) there are two types of restriction on the triangulations; One by $\zeta_{n, N}$, that measures the quasiuniformity of the family of triangulation, and the other by $\delta_{\mathcal{F}_{h}}$, that measure the regularity of the family of triangulations in a slightly different sense. Although maybe not explicitly, but $\zeta_{n, N}$ and $\delta_{\mathcal{F}_{h}}$ can be related. In practice we use finitely many triangulations, that means quasiuniformity holds, though possibly with big $\zeta_{n, N}$, see Remark 2 This means that we still can use the a posteriori error estimate (5.6). But when $\delta_{\mathcal{F}_{h}}$ is not sufficiently small, the error estimate (5.6) does not hold. This calls for using local interpolants instead of global $L_{2}$-projection $\mathcal{P}_{h}$. In the next section we present an a posteriori error estimate using interpolation, both in space and time, on each space-time cell.

\section{A posteriori error estimates based on local projections}

We recall the decomposition of the space-time slab $\Omega^{n}=\Omega \times I_{n}$ into cells $K^{n}=$ $K \times I_{n}, K \in \overline{\mathcal{T}}_{h}^{n}$. Let $I_{h k}$ be a standard interpolant, for example linear in space and constant in time, such that the following error estimates hold for the error operator $E_{h k} v=\left(I_{h k}-I\right) v$.

$$
\begin{aligned}
\left\|E_{h k} v\right\|_{K^{n}} & \leq C\left(h_{K}^{r}\left\|\nabla^{r} v\right\|_{K^{n}}+k_{n}\|\dot{v}\|_{K^{n}}\right), \quad r=1,2, \\
\left\|\nabla E_{h k} v\right\|_{K^{n}} & \leq C\left(h_{K}\left\|\nabla^{2} v\right\|_{K^{n}}+k_{n}\|\nabla \dot{v}\|_{K^{n}}\right) .
\end{aligned}
$$

We recall the mesh function $\bar{h}_{n}$ from (3.2), and $\bar{h}_{\max , n}=\max _{K \in \overline{\mathcal{T}}_{h}^{n}} \bar{h}_{K}$. We will also use the fact that

$$
\|v\|_{\Omega^{n}}^{2}=\int_{I_{n}}\|v(t)\|^{2} d t \leq k_{n} \max _{I_{n}}\|v(t)\|^{2} .
$$

Theorem 3. Let $u$ and $U$ be the solutions of (2.6) and (3.4), respectively, and we assume that $\mathcal{K} \in L_{2}\left(\mathbb{R}^{+}\right)$. Then, with $e=U-u$, we have the weighted a posteriori 
error estimate, for $\alpha=1,2$,

$$
\begin{aligned}
\left|L^{*}(e)\right| \leq & C \max _{[0, T]}\left\{\left\|\nabla^{\alpha} z_{1}(t)\right\|,\left\|\nabla^{2} z_{2}(t)\right\|,\left\|\dot{z}_{1}(t)\right\|,\left\|\dot{z}_{2}(t)\right\|,\left\|\nabla \dot{z}_{2}(t)\right\|\right\} \\
& \times\left\{\Upsilon_{0}+\sum_{n=1}^{N}\left(\Upsilon_{n, 1}+\Upsilon_{n, 2}\right)\right\} .
\end{aligned}
$$

where

$$
\begin{aligned}
\Upsilon_{0}= & \left\|h_{0}^{\alpha}\left(U_{1}(0)-u^{0}\right)\right\|+\left\|h_{0}^{2}\left(U_{2}(0)-v^{0}\right)\right\|, \\
\Upsilon_{n, 1} & =k_{n}^{1 / 2}\left\{\left\|\bar{h}_{n}^{\alpha}\left(\dot{U}_{1}-U_{2}\right)\right\|_{\Omega^{n}}+k_{n}\left\|\dot{U}_{1}-U_{2}\right\|_{\Omega^{n}}\right. \\
& +\left\|\bar{h}_{n}^{2}\left(\dot{U}_{2}-f\right)\right\|_{\Omega^{n}}+k_{n}\left\|\dot{U}_{2}-f\right\|_{\Omega^{n}} \\
& +\left(\sum_{K \in \overline{\mathcal{T}}_{h}^{n}} \bar{h}_{K}^{3}\left\|r_{d}\right\|_{\partial K^{n}}^{2}\right)^{1 / 2}+\left(\sum_{K \in \overline{\mathcal{T}}_{h}^{n}} \bar{h}_{K}^{3}\left\|g_{h}-g\right\|_{\partial K^{n}}^{2}\right)^{1 / 2} \\
& +k_{n}\left(\sum_{K \in \overline{\mathcal{T}}_{h}^{n}} \bar{h}_{K}^{-1}\left\|r_{d}\right\|_{\partial K^{n}}^{2}\right)^{1 / 2}+k_{n}\left(\sum_{K \in \overline{\mathcal{T}}_{h}^{n}} \bar{h}_{K}^{-1}\left\|g_{h}-g\right\|_{\partial K^{n}}^{2}\right)^{1 / 2} \\
& \left.+k_{n}\left(\sum_{K \in \overline{\mathcal{T}}_{h}^{n}} \bar{h}_{K}\left\|r_{d}\right\|_{\partial K^{n}}^{2}\right)^{1 / 2}+k_{n}\left(\sum_{K \in \overline{\mathcal{T}}_{h}^{n}} \bar{h}_{K}\left\|g_{h}-g\right\|_{\partial K^{n}}^{2}\right)^{1 / 2}\right\}, \\
& \Upsilon_{\Upsilon_{n}} \sum_{j=n}^{N} k_{j}^{1 / 2}\|\mathcal{K}\|_{L_{2}\left(I_{j}\right)}\left\{\left(\sum_{K \in \overline{\mathcal{T}}_{h}^{j}} \bar{h}_{K}^{3}\left\|r_{d}(t)\right\|_{\partial K}^{2}\right)^{1 / 2}\right. \\
& \left.+k_{j}\left(\sum_{K \in \overline{\mathcal{T}}_{h}^{j}} \bar{h}_{K}^{-1}\left\|r_{d}(t)\right\|_{\partial K}^{2}\right)^{1 / 2}+k_{j}\left(\sum_{K \in \overline{\mathcal{T}}_{h}^{j}} \bar{h}_{K}\left\|r_{d}(t)\right\|_{\partial K}^{2}\right)^{1 / 2}\right\} d t .
\end{aligned}
$$

Proof. We write the error representation (4.3) as

$$
L^{*}(e)=\sum_{K \in \mathcal{T}_{h}^{0}} \Theta_{0, K}+\sum_{i=1}^{5} \sum_{n=1}^{N} \sum_{K \in \overline{\mathcal{T}}_{h}^{n}} \Theta_{i, K}^{n}=\mathbf{I}_{0}+\sum_{i=1}^{5} \mathbf{I}_{i} .
$$

First we estimate $\mathbf{I}_{0}$. To this end, recalling $\Theta_{0, K}$ from (4.5), we use the CauchySchwarz inequality and the interpolation error estimate (6.1) to obtain, for $\alpha=1,2$,

$$
\begin{aligned}
\sum_{K \in \mathcal{T}_{h}^{0}}\left(U_{1}(0)-u^{0}, E_{h k} z_{1}(0)\right)_{K} & \leq \sum_{K \in \mathcal{T}_{h}^{0}}\left\|U_{1}(0)-u^{0}\right\|_{K}\left\|E_{h k} z_{1}(0)\right\|_{K} \\
& \leq C \sum_{K \in \mathcal{T}_{h}^{0}}\left\|U_{1}(0)-u^{0}\right\|_{K} h_{K}^{\alpha}\left\|\nabla^{\alpha} z_{1}(0)\right\|_{K} \\
& \leq C\left\|\nabla^{\alpha} z_{1}(0)\right\|\left(\sum_{K \in \mathcal{T}_{h}^{0}} h_{K}^{2 \alpha}\left\|U_{1}(0)-u^{0}\right\|_{K}^{2}\right)^{1 / 2} \\
& =C\left\|\nabla^{\alpha} z_{1}(0)\right\|\left\|h_{0}^{\alpha}\left(U_{1}(0)-u^{0}\right)\right\| .
\end{aligned}
$$

Similarly we have

$$
\sum_{K \in \mathcal{T}_{h}^{0}}\left(U_{2}(0)-v^{0}, E_{h k} z_{2}(0)\right)_{K} \leq C\left\|\nabla^{2} z_{2}(0)\right\|\left\|h_{0}^{2}\left(U_{2}(0)-v^{0}\right)\right\| .
$$


From these two estimates we conclude

(6.6) $\mathbf{I}_{0} \leq C \max \left\{\left\|\nabla^{\alpha} z_{1}(0)\right\|,\left\|\nabla^{2} z_{2}(0)\right\|\right\}\left\{\left\|h_{0}^{\alpha}\left(U_{1}(0)-u^{0}\right)\right\|+\left\|h_{0}^{2}\left(U_{2}(0)-v^{0}\right)\right\|\right\}$.

For the next term, using the Cauchy-Schwarz inequality and the error estimate (6.1), we have, for $\alpha=1,2$,

$$
\begin{aligned}
\mathbf{I}_{1} \leq & \sum_{n=1}^{N} \sum_{K \in \overline{\mathcal{T}}_{h}^{n}}\left\|\dot{U}_{1}-U_{2}\right\|_{K^{n}}\left\|E_{h k} z_{1}\right\|_{K^{n}} \\
\leq & C \sum_{n=1}^{N} \sum_{K \in \overline{\mathcal{T}}_{h}^{n}}\left\|\dot{U}_{1}-U_{2}\right\|_{K^{n}}\left(\bar{h}_{K}^{\alpha}\left\|\nabla^{\alpha} z_{1}\right\|_{K^{n}}+k_{n}\left\|\dot{z}_{1}\right\|_{K^{n}}\right) \\
\leq & C \sum_{n=1}^{N}\left(\sum_{K \in \overline{\mathcal{T}}_{h}^{n}} \bar{h}_{K}^{2 \alpha}\left\|\dot{U}_{1}-U_{2}\right\|_{K^{n}}^{2}\right)^{1 / 2}\left(\sum_{K \in \overline{\mathcal{T}}_{h}^{n}}\left\|\nabla^{\alpha} z_{1}\right\|_{K^{n}}^{2}\right)^{1 / 2} \\
& +C \sum_{n=1}^{N} k_{n}\left(\sum_{K \in \overline{\mathcal{T}}_{h}^{n}}\left\|\dot{U}_{1}-U_{2}\right\|_{K^{n}}^{2}\right)^{1 / 2}\left(\sum_{K \in \overline{\mathcal{T}}_{h}^{n}}\left\|\dot{z}_{1}\right\|_{K^{n}}^{2}\right)^{1 / 2} \\
\leq & C \sum_{n=1}^{N}\left\{\left\|\bar{h}_{n}^{\alpha}\left(\dot{U}_{1}-U_{2}\right)\right\|_{\Omega^{n}}\left\|\nabla^{\alpha} z_{1}\right\|_{\Omega^{n}}+k_{n}\left\|\dot{U}_{1}-U_{2}\right\|_{\Omega^{n}}\left\|\dot{z}_{1}\right\|_{\Omega^{n}}\right\}
\end{aligned}
$$

that using (6.3) we have

$$
\mathbf{I}_{1} \leq C \max _{[0, T]}\left\{\left\|\nabla^{\alpha} z_{1}(t)\right\|,\left\|\dot{z}_{1}(t)\right\|\right\} \sum_{n=1}^{N} k_{n}^{1 / 2}\left\{\left\|\bar{h}_{n}^{\alpha}\left(\dot{U}_{1}-U_{2}\right)\right\|_{\Omega^{n}}+k_{n}\left\|\dot{U}_{1}-U_{2}\right\|_{\Omega^{n}}\right\} .
$$

In the same way we obtain

$$
\mathbf{I}_{2} \leq C \max _{[0, T]}\left\{\left\|\nabla^{2} z_{2}(t)\right\|,\left\|\dot{z}_{2}(t)\right\|\right\} \sum_{n=1}^{N} k_{n}^{1 / 2}\left\{\left\|\bar{h}_{n}^{2}\left(\dot{U}_{2}-f\right)\right\|_{\Omega^{n}}+k_{n}\left\|\dot{U}_{2}-f\right\|_{\Omega^{n}}\right\} .
$$


Now for $\mathbf{I}_{3}$, we use the Cauchy-Schwarz inequality, the scaled trace inequality (5.5), and the error estimates (6.1) - 6.2 to obtain,

$$
\begin{aligned}
\mathbf{I}_{3} \leq & \sum_{n=1}^{N} \sum_{K \in \overline{\mathcal{T}}_{h}^{n}}\left\|g_{d}-g\right\|_{\partial K^{n}}\left\|E_{h k} z_{2}\right\|_{\partial K^{n}} \\
\leq & C \sum_{n=1}^{N} \sum_{K \in \overline{\mathcal{T}}_{h}^{n}}\left\|g_{d}-g\right\|_{\partial K^{n}}\left\{\bar{h}_{K}^{-1 / 2}\left\|E_{h k} z_{2}\right\|_{K^{n}}+\bar{h}_{K}^{1 / 2}\left\|\nabla E_{h k} z_{2}\right\|_{K^{n}}\right\} \\
\leq & C \sum_{n=1}^{N} \sum_{K \in \overline{\mathcal{T}}_{h}^{n}}\left\|g_{d}-g\right\|_{\partial K^{n}} \\
& \times\left\{2 \bar{h}_{K}^{3 / 2}\left\|\nabla^{2} z_{2}\right\|_{K^{n}}+\bar{h}_{K}^{-1 / 2} k_{n}\left\|\dot{z}_{2}\right\|_{K^{n}}+\bar{h}_{K}^{1 / 2} k_{n}\left\|\nabla \dot{z}_{2}\right\|_{K^{n}}\right\} \\
\leq & C \sum_{n=1}^{N}\left\{\left(\sum_{K \in \overline{\mathcal{T}}_{h}^{n}} \bar{h}_{K}^{3}\left\|g_{d}-g\right\|_{\partial K^{n}}^{2}\right)^{1 / 2}\left\|\nabla^{2} z_{2}\right\|_{\Omega^{n}}\right. \\
& +k_{n}\left(\sum_{K \in \overline{\mathcal{T}}_{h}^{n}} \bar{h}_{K}^{-1}\left\|g_{d}-g\right\|_{\partial K^{n}}^{2}\right)^{1 / 2}\left\|\dot{z}_{2}\right\|_{\Omega^{n}} \\
& \left.+k_{n}\left(\sum_{K \in \overline{\mathcal{T}}_{h}^{n}} \bar{h}_{K}\left\|g_{d}-g\right\|_{\partial K^{n}}^{2}\right)^{1 / 2}\left\|\nabla \dot{z}_{2}\right\|_{\Omega^{n}}\right\},
\end{aligned}
$$

that using (6.3) we have

$$
\begin{aligned}
\mathbf{I}_{3} \leq & C \max _{[0, T]}\left\{\left\|\nabla^{2} z_{2}(t)\right\|,\left\|\dot{z}_{2}(t)\right\|,\left\|\nabla \dot{z}_{2}(t)\right\|\right\} \\
& \times \sum_{n=1}^{N} k_{n}^{1 / 2}\left\{\left(\sum_{K \in \overline{\mathcal{T}}_{h}^{n}} \bar{h}_{K}^{3}\left\|g_{d}-g\right\|_{\partial K^{n}}^{2}\right)^{1 / 2}+k_{n}\left(\sum_{K \in \overline{\mathcal{T}}_{h}^{n}} \bar{h}_{K}^{-1}\left\|g_{d}-g\right\|_{\partial K^{n}}^{2}\right)^{1 / 2}\right. \\
& \left.+k_{n}\left(\sum_{K \in \overline{\mathcal{T}}_{h}^{n}} \bar{h}_{K}\left\|g_{d}-g\right\|_{\partial K^{n}}^{2}\right)^{1 / 2}\right\}
\end{aligned}
$$

And similarly

$$
\begin{aligned}
\mathbf{I}_{4} \leq & C \max _{[0, T]}\left\{\left\|\nabla^{2} z_{2}(t)\right\|,\left\|\dot{z}_{2}(t)\right\|,\left\|\nabla \dot{z}_{2}(t)\right\|\right\} \\
& \times \sum_{n=1}^{N} k_{n}^{1 / 2}\left\{\left(\sum_{K \in \overline{\mathcal{T}}_{h}^{n}} \bar{h}_{K}^{3}\left\|r_{d}\right\|_{\partial K^{n}}^{2}\right)^{1 / 2}+k_{n}\left(\sum_{K \in \overline{\mathcal{T}}_{h}^{n}} \bar{h}_{K}^{-1}\left\|r_{d}\right\|_{\partial K^{n}}^{2}\right)^{1 / 2}\right. \\
& \left.\quad+k_{n}\left(\sum_{K \in \overline{\mathcal{T}}_{h}^{n}} \bar{h}_{K}\left\|r_{d}\right\|_{\partial K^{n}}^{2}\right)^{1 / 2}\right\} .
\end{aligned}
$$


Finally we find an estimate for $\mathbf{I}_{5}$ which includes the convolution terms. First, recalling the definition of $\Theta_{5, K}^{n}$ from (4.5), we can write $\mathbf{I}_{5}$ as,

$$
\begin{aligned}
\mathbf{I}_{5} & =-\sum_{n=1}^{N} \int_{I_{n}} \int_{s}^{T} \mathcal{K}(s-t) \sum_{K \in \overline{\mathcal{T}}_{h}^{n}}\left(r_{d}(t), E_{h k} z_{2}(s)\right)_{\partial K} d s d t \\
& =-\sum_{n=1}^{N} \int_{I_{n}} \sum_{j=n}^{N} \int_{t \vee t_{j-1}}^{t_{j}} \mathcal{K}(s-t) \sum_{K \in \overline{\mathcal{T}}_{h}^{j}}\left(r_{d}(t), E_{h k} z_{2}(s)\right)_{\partial K} d s d t .
\end{aligned}
$$

Then, using the Cauchy-Schwarz inequality and the assumption $\mathcal{K} \in L_{2}\left(\mathbb{R}^{+}\right)$, we have

$$
\begin{aligned}
\left|\mathbf{I}_{5}\right| & \leq \sum_{n=1}^{N} \int_{I_{n}} \sum_{j=n}^{N} \sum_{K \in \overline{\mathcal{T}}_{h}^{j}}\left\|r_{d}(t)\right\|_{\partial K} \int_{t \vee t_{j-1}}^{t_{j}} \mathcal{K}(s-t)\left\|E_{h k} z_{2}(s)\right\|_{\partial K} d s d t \\
\leq & \sum_{n=1}^{N} \int_{I_{n}} \sum_{j=n}^{N} \sum_{K \in \overline{\mathcal{T}}_{h}^{j}}\left\|r_{d}(t)\right\|_{\partial K}\left(\int_{t \vee t_{j-1}}^{t_{j}} \mathcal{K}^{2}(s-t) d s\right)^{1 / 2} \\
& \times\left(\int_{t \vee t_{j-1}}^{t_{j}}\left\|E_{h k} z_{2}(s)\right\|_{\partial K}^{2} d s\right)^{1 / 2} d t \\
& \leq \sum_{n=1}^{N} \int_{I_{n}} \sum_{j=n}^{N} \sum_{K \in \overline{\mathcal{T}}_{h}^{j}}\left\|r_{d}(t)\right\|_{\partial K} d t\|\mathcal{K}\|_{L_{2}\left(I_{j}\right)}\left\|E_{h k} z_{2}(s)\right\|_{\partial K^{j}},
\end{aligned}
$$

that, using the scaled trace inequality (5.5), the error estimates (6.1)- (6.2), and the Cauchy-Schwarz inequality over the triangles, we have

$$
\begin{aligned}
\left|\mathbf{I}_{5}\right| \leq C & \sum_{n=1}^{N} \int_{I_{n}} \sum_{j=n}^{N}\|\mathcal{K}\|_{L_{2}\left(I_{j}\right)}\left\{\left(\sum_{K \in \overline{\mathcal{T}}_{h}^{j}} \bar{h}_{K}^{3}\left\|r_{d}(t)\right\|_{\partial K}^{2}\right)^{1 / 2}\left\|\nabla^{2} z_{2}(s)\right\|_{\Omega^{j}}\right. \\
& +k_{j}\left(\sum_{K \in \overline{\mathcal{T}}_{h}^{j}} \bar{h}_{K}^{-1}\left\|r_{d}(t)\right\|_{\partial K}^{2}\right)^{1 / 2}\left\|\dot{z}_{2}(s)\right\|_{\Omega^{j}} \\
& \left.+k_{j}\left(\sum_{K \in \overline{\mathcal{T}}_{h}^{j}} \bar{h}_{K}\left\|r_{d}(t)\right\|_{\partial K}^{2}\right)^{1 / 2}\left\|\nabla \dot{z}_{2}(s)\right\|_{\Omega^{j}}\right\} d t
\end{aligned}
$$

Hence, by (6.3), we conclude

$$
\begin{aligned}
\left|\mathbf{I}_{5}\right| \leq C \max _{[0, T]}\{ & \left.\left\|\nabla^{2} z_{2}(t)\right\|,\left\|\dot{z}_{2}(t)\right\|,\left\|\nabla \dot{z}_{2}(t)\right\|\right\} \sum_{n=1}^{N} \int_{I_{n}} \sum_{j=n}^{N}\|\mathcal{K}\|_{L_{2}\left(I_{j}\right)} k_{j}^{\frac{1}{2}} \\
\times & \left\{\left(\sum_{K \in \overline{\mathcal{T}}_{h}^{j}} \bar{h}_{K}^{3}\left\|r_{d}(t)\right\|_{\partial K}^{2}\right)^{1 / 2}+k_{j}\left(\sum_{K \in \overline{\mathcal{T}}_{h}^{j}} \bar{h}_{K}^{-1}\left\|r_{d}(t)\right\|_{\partial K}^{2}\right)^{1 / 2}\right. \\
& \left.+k_{j}\left(\sum_{K \in \overline{\mathcal{T}}_{h}^{j}} \bar{h}_{K}\left\|r_{d}(t)\right\|_{\partial K}^{2}\right)^{1 / 2}\right\} d t .
\end{aligned}
$$

Putting estimates (6.6) 6 (6.11) in we conclude the a posteriori error estimate (6.4). Now the proof is complete. 
Remark 4. We note that, for example, we can compute $\left\|r_{d}\right\|_{\partial K^{n}}$ as

$$
\begin{aligned}
\left\|r_{d}\right\|_{\partial K^{n}} & =\left(\int_{I_{n}}\left\|\frac{t-t_{n}}{-k_{n}} r_{d}\left(t_{n-1}\right)+\frac{t-t_{n-1}}{k_{n}} r_{d}\left(t_{n}\right)\right\|_{\partial K}^{2} d t\right)^{1 / 2} \\
& \leq \frac{k_{n}^{1 / 2}}{\sqrt{3}}\left(\left\|r_{d}\left(t_{n-1}\right)\right\|_{\partial K}^{2}+\left\|r_{d}\left(t_{n}\right)\right\|_{\partial K}^{2}\right)^{1 / 2} \\
& \leq \sqrt{\frac{2}{3}} k_{n}^{1 / 2}\left(\left\|r_{d}\left(t_{n-1}\right)\right\|_{\partial K}+\left\|r_{d}\left(t_{n}\right)\right\|_{\partial K}\right) .
\end{aligned}
$$

Remark 5. We recall that to prove the a posteriori error estimate (6.4), we used the second error representaion (4.3) in (6.5). Therefore, the error indicator $\Upsilon_{n, 2}$ is not space-time cellwise, since the convolution integral applies to the error term $E_{h k} z_{2}$. Besides, we need the assumption $\mathcal{K} \in L_{2}\left(\mathbb{R}^{+}\right)$for the kernel, that does not apply to weakly singular kernels for which we have $\mathcal{K} \in L_{1}\left(\mathbb{R}^{+}\right)$. Hence for this case, when $\mathcal{K} \in L_{1}\left(\mathbb{R}^{+}\right)$, the error indicator $\Upsilon_{n, 2}$ is not fulfilled, and we need to use the first error representation (4.2) in (6.5). Now we just need to estimate the new $\mathbf{I}_{5}$, that using the notation (4.1) is writen in the form

$$
\begin{aligned}
\mathbf{I}_{5} & =-\sum_{n=1}^{N} \int_{I_{n}} \sum_{j=1}^{n} \sum_{K \in \overline{\mathcal{T}}_{h}^{j}}\left(\int_{t_{j-1}}^{t \wedge t_{j}} \mathcal{K}(t-s) r_{d}(s) d s, E_{h k} z_{2}(t)\right)_{\partial K} d t \\
& =-\sum_{n=1}^{N} \int_{I_{n}} \sum_{j=1}^{n} \sum_{K \in \overline{\mathcal{T}}_{h}^{j}}\left(\left(\mathcal{K} * r_{d}\right)^{j}(t), E_{h k} z_{2}(t)\right)_{\partial K} d t .
\end{aligned}
$$


Then, by the Cauchy-Schwarz inequality and the scaled trace inequality (5.5), we have

$$
\begin{aligned}
\left|\mathbf{I}_{5}\right| \leq & C \sum_{n=1}^{N} \int_{I_{n}} \sum_{j=1}^{n}\left\{\left(\sum_{K \in \overline{\mathcal{T}}_{h}^{j}} \bar{h}_{K}^{-1}\left\|\left(\mathcal{K} * r_{d}\right)^{j}(t)\right\|_{\partial K}^{2}\right)^{1 / 2}\right. \\
& \left.\times\left(\sum_{K \in \overline{\mathcal{T}}_{h}^{j}} \bar{h}_{K}\left\|E_{h k} z_{2}(t)\right\|_{\partial K}^{2}\right)^{1 / 2}\right\} d t \\
\leq & C \sum_{n=1}^{N} \int_{I_{n}} \sum_{j=1}^{n}\left\{\left(\sum_{K \in \overline{\mathcal{T}}_{h}^{j}} \bar{h}_{K}^{-1}\left\|\left(\mathcal{K} * r_{d}\right)^{j}(t)\right\|_{\partial K}^{2}\right)^{1 / 2}\right. \\
& \left.\times\left(\sum_{K \in \overline{\mathcal{T}}_{h}^{j}}\left\|E_{h k} z_{2}(t)\right\|_{K}^{2}+\bar{h}_{K}^{2}\left\|\nabla E_{h k} z_{2}(t)\right\|_{K}^{2}\right)^{1 / 2}\right\} d t \\
\leq & C \sum_{n=1}^{N} \int_{I_{n}} \sum_{j=1}^{n}\left\{\left(\sum_{K \in \overline{\mathcal{T}}_{h}^{j}} \bar{h}_{K}^{-1}\left\|\left(\mathcal{K} * r_{d}\right)^{j}(t)\right\|_{\partial K}^{2}\right)^{1 / 2}\right. \\
& \left.\times\left(\left\|E_{h k} z_{2}(t)\right\|+\bar{h}_{m a x, j}\left\|\nabla E_{h k} z_{2}(t)\right\|\right)\right\} d t \\
= & C \sum_{n=1}^{N} \int_{I_{n}}\left\|E_{h k} z_{2}(t)\right\| \sum_{j=1}^{n}\left(\sum_{K \in \overline{\mathcal{T}}_{h}^{j}} \bar{h}_{K}^{-1}\left\|\left(\mathcal{K} * r_{d}\right)^{j}(t)\right\|_{\partial K}^{2}\right)^{1 / 2} d t \\
+ & C \sum_{n=1}^{N} \int_{I_{n}}\left\|\nabla E_{h k} z_{2}(t)\right\| \sum_{j=1}^{n} \bar{h}_{m a x, j}\left(\sum_{K \in \overline{\mathcal{T}}_{h}^{j}} \bar{h}_{K}^{-1}\left\|\left(\mathcal{K} * r_{d}\right)^{j}(t)\right\|_{\partial K}^{2}\right)^{1 / 2} d t
\end{aligned}
$$

and using the Cauchy-Schwarz inequality in the integrals over $I_{n}$, we have

$$
\begin{aligned}
\left|\mathbf{I}_{5}\right| \leq C & \sum_{n=1}^{N}\left\|E_{h k} z_{2}\right\|_{\Omega^{n}}\left(\int_{I_{n}}\left(\sum_{j=1}^{n}\left(\sum_{K \in \overline{\mathcal{T}}_{h}^{j}} \bar{h}_{K}^{-1}\left\|\left(\mathcal{K} * r_{d}\right)^{j}(t)\right\|_{\partial K}^{2}\right)^{1 / 2}\right)^{2} d t\right)^{1 / 2} \\
+ & C \sum_{n=1}^{N}\left\|\nabla E_{h k} z_{2}\right\|_{\Omega^{n}} \\
& \times\left(\int_{I_{n}}\left(\sum_{j=1}^{n} \bar{h}_{\text {max }, j}\left(\sum_{K \in \overline{\mathcal{T}}_{h}^{j}} \bar{h}_{K}^{-1}\left\|\left(\mathcal{K} * r_{d}\right)^{j}(t)\right\|_{\partial K}^{2}\right)^{1 / 2}\right)^{2} d t\right)^{1 / 2}
\end{aligned}
$$

Since by the error estimate (6.1) we have

$$
\begin{aligned}
\int_{I_{n}}\left\|E_{h k} z_{2}(t)\right\|^{2} d t & =\int_{I_{n}} \sum_{K \in \overline{\mathcal{T}}_{h}^{n}}\left\|E_{h k} z_{2}(t)\right\|_{K}^{2} d t=\sum_{K \in \overline{\mathcal{T}}_{h}^{n}}\left\|E_{h k} z_{2}(t)\right\|_{K^{n}}^{2} \\
& \leq C \sum_{K \in \overline{\mathcal{T}}_{h}^{n}}\left(\bar{h}_{K}^{4}\left\|\nabla^{2} z_{2}\right\|_{K^{n}}^{2}+k_{n}^{2}\left\|\dot{z}_{2}\right\|_{K^{n}}^{2}\right) \\
& \leq C\left(\bar{h}_{\max , n}^{4}\left\|\nabla^{2} z_{2}\right\|_{\Omega^{n}}^{2}+k_{n}^{2}\left\|\dot{z}_{2}\right\|_{\Omega^{n}}^{2}\right)
\end{aligned}
$$


and similarly by (6.2) we have

$$
\begin{aligned}
\int_{I_{n}}\left\|\nabla E_{h k} z_{2}(t)\right\|^{2} d t & =\int_{I_{n}} \sum_{K \in \overline{\mathcal{T}}_{h}^{n}}\left\|\nabla E_{h k} z_{2}(t)\right\|_{K}^{2} d t=\sum_{K \in \overline{\mathcal{T}}_{h}^{n}}\left\|\nabla E_{h k} z_{2}(t)\right\|_{K^{n}}^{2} \\
& \leq C \sum_{K \in \overline{\mathcal{T}}_{h}^{n}}\left(\bar{h}_{K}^{2}\left\|\nabla^{2} z_{2}\right\|_{\tilde{K}^{n}}^{2}+k_{n}^{2}\left\|\nabla \dot{z}_{2}\right\|_{\tilde{K}^{n}}^{2}\right) \\
& \leq C\left(\bar{h}_{\max , n}^{2}\left\|\nabla^{2} z_{2}\right\|_{\Omega^{n}}^{2}+k_{n}^{2}\left\|\nabla \dot{z}_{2}\right\|_{\Omega^{n}}^{2}\right),
\end{aligned}
$$

then, recalling (6.3), we conclude the estimate

$$
\begin{aligned}
\mathbf{I}_{5} \leq C \max _{[0, T]} & \left\{\left\|\nabla^{2} z_{2}(t)\right\|,\left\|\dot{z}_{2}(t)\right\|,\left\|\nabla \dot{z}_{2}(t)\right\|\right\}\left\{\sum_{n=1}^{N} k_{n}^{1 / 2}\left(\bar{h}_{\text {max }, n}^{2}+k_{n}\right)\right. \\
& \times\left(\int_{I_{n}}\left(\sum_{j=1}^{n}\left(\sum_{K \in \overline{\mathcal{T}}_{h}^{j}} \bar{h}_{K}^{-1}\left\|\left(\mathcal{K} * r_{d}\right)^{j}(t)\right\|_{\partial K}^{2}\right)^{1 / 2}\right)^{2} d t\right)^{1 / 2} \\
+ & C \sum_{n=1}^{N} k_{n}^{1 / 2}\left(\bar{h}_{\max , n}+k_{n}\right) \\
& \left.\times\left(\int_{I_{n}}\left(\sum_{j=1}^{n} \bar{h}_{\max , j}\left(\sum_{K \in \overline{\mathcal{T}}_{h}^{j}} \bar{h}_{K}^{-1}\left\|\left(\mathcal{K} * r_{d}\right)^{j}(t)\right\|_{\partial K}^{2}\right)^{1 / 2}\right)^{2} d t\right)^{1 / 2}\right\} .
\end{aligned}
$$

This means that, for the case $\mathcal{K} \in L_{1}\left(\mathbb{R}^{+}\right)$, the error indicator $\Upsilon_{n, 2}$ in the a posteriori error estimate (6.4) is

$$
\begin{aligned}
& k_{n}^{1 / 2}\left\{\left(\bar{h}_{\text {max }, n}^{2}+k_{n}\right)\left(\int_{I_{n}}\left(\sum_{j=1}^{n}\left(\sum_{K \in \overline{\mathcal{T}}_{h}^{j}} \bar{h}_{K}^{-1}\left\|\left(\mathcal{K} * r_{d}\right)^{j}(t)\right\|_{\partial K}^{2}\right)^{1 / 2}\right)^{2} d t\right)^{1 / 2}\right. \\
& \left.\quad+\left(\bar{h}_{\text {max }, n}+k_{n}\right)\left(\int_{I_{n}}\left(\sum_{j=1}^{n} \bar{h}_{\text {max }, j}\left(\sum_{K \in \overline{\mathcal{T}}_{h}^{j}} \bar{h}_{K}^{-1}\left\|\left(\mathcal{K} * r_{d}\right)^{j}(t)\right\|_{\partial K}^{2}\right)^{1 / 2}\right)^{2} d t\right)^{1 / 2}\right\} .
\end{aligned}
$$

We note that the last a posteriori error estimate presented in (6.4), does not have the restrictions that were mentioned in Remark 3

\section{Conclusion}

In this work, a space-time continuous Galerkin finite element method has been applied to a hyperbolic type integro-differential equation. To provide the main tools for adaptive strategies, mainly based on the DWR approach, we have presented three error representations in Theorem 1. The main difference between these a posteriori error representations is that we apply the convolution integral either on the residual $r_{d}$ or on the error term $E_{h k} z_{2}$. Obviously we choose the cheaper one, depending on the method we choose for computing or estimating $E_{h k} z_{2}$.

Evaluation of a posteriori error representations require information about the dual solution. One way is to find bounds for the dual solution in certain Sobolev norms, that can be estimated, e.g., by means of stability estimates. In Theorem 2 we have presented a weighted global a posteriori error estimate, that is used when the goal functional is global. The error indicators have been separated, such that 
it can be used as a basis for independent adaptation of the time step and spatial mesh. However, for this global error estimate, the triangulations are required to be quasiuniform and also fulfill a certain kind of regularity, see Remark 3. This restriction and also when we use local goal functionals call for local projections. In Theorem 3 standard local projections have been used to obtain a posteriori error estimates.

For adaptive strategies the error representations can be used to accurately estimate the actual errors for getting a stopping criterion of the adaptation process. Then we can use the global/local a posteriori error estimates for steering the adaptation process.

Sparse quadrature can be used to overcome the problem with the growing amount of data, in the convolution term, that has to be stored and used in time stepping methods. However, we note that this is not an issue for exponentially decaying memory kernels, in linear viscoelasticity, that are represented as a Prony series. This is due to the existence of a recurrence formula for history updating. We plan to address adaptivity strategies based on sparse quadrature together with numerical adaptation methods such as the DWR approach, using the theory presented here, in future work.

Acknowledgment. I would like thank Prof. Stig Larsson for helpful discussion and constructive comments.

\section{REFERENCES}

1. K. Adolfsson, M. Enelund, and S. Larsson, Adaptive discretization of an integro-differential equation with a weakly singular convolution kernel, Comput. Methods Appl. Mech. Engrg. 192 (2003), 5285-5304.

2. _ Adaptive discretization of fractional order viscoelasticity using sparse time history, Comput. Methods Appl. Mech. Engrg. 193 (2004), 4567-4590.

3. K. Adolfsson, M. Enelund, and S. Larsson, Space-time discretization of an integro-differential equation modeling quasi-static fractional-order viscoelasticity, J. Vib. Control 14 (2008), $1631-1649$.

4. K. Adolfsson, M. Enelund, S. Larsson, and M. Racheva, Discretization of integro-differential equations modeling dynamic fractional order viscoelasticity, LNCS 3743 (2006), 76-83.

5. W. Bangerth, M. Geiger, and R. Rannacher, Adaptive Galerkin finite element methods for the wave equation, CMAM. 10 (2010), 3-48.

6. W. Bangerth and R. Rannacher, Adaptive Finite Element Methods for Differential Equations, Lectures in Mathematics ETH Zürich, Birkhäuser Verlag, 2003.

7. M. Boman, Estimates for the $L_{2}$-projection onto continuous finite element spaces in a weighted $L_{p}$-norm, BIT 46 (2006), 249-260.

8. K. Eriksson, D. Estep, P. Hansbo, and C. Johnson, Introduction to adaptive methods for differential equations, Acta Numerica 4 (1995), 105-158.

9. C. Johnson, Discontinuous Galerkin finite element methods for second order hyperbolic problems, Comput. Methods Appl. Mech. Engrg. 107 (1993), 117-129.

10. M. Karamanou, S. Shaw, M. K. Warby, and J. R. Whiteman, Models, algorithms and error estimation for computational viscoelasticity, Comput. Methods Appl. Mech. Engrg. 194 (2005), 245-265.

11. S. Larsson and F. Saedpanah, The continuous Galerkin method for an integro-differential equation modeling dynamic fractional order viscoelasticity, IMA J. Numer. Anal. 30 (2010), 964-986.

12. C. Lubich, Convolution quadrature and discretized operational calculus I, Numeriche Mathematik 52 (1988), 129-145.

13. Ch. Lubich, I. H. Sloan, and V. Thomée, Nonsmooth data error estimates for approximations of an evolution equation with a positive-type memory term, Math. Comp. 65 (1996), 1-17. 
14. W. McLean and V. Thomée, Numerical solution via Laplace transforms of a fractional order evolution equation, J. Integral Equations Appl. 22 (2010), 57-94.

15. W. McLean, V. Thomée, and L. B. Wahlbin, Discretization with variable time steps of an evolution equation with a positive-type memory term, J. of Comput. Appl. Math. 69 (1996), 49-69.

16. J. E. M. Rivera and G. P. Menzala, Decay rates of solution to a von Kármán system for viscoelastic plates with memory, Quart. Appl. Math. Eng. LVII (1999), 181-200.

17. B. Rivière, S. Shaw, and J. R. Whiteman, Discontinuous Galerkin finite element methods for dynamic linear solid viscoelasticity problems, Numer. Methods Partial Differential Equations 23 (2007), 1149-1166.

18. F. Saedpanah, Well-posedness of an integro-differential equation with positive type kernels modeling fractional order viscoelasticity, Cornell University Library, arXiv:1203.4001.

19. A. Schädle, M. López-Fernández, and Ch. Lubich, Adaptive, fast, and oblivious convolution in evolution equations with memory, SIAM J. Sci. Comput. 30 (2008), 1015-1037.

20. S. Shaw, M. K. Warby, and J. R. Whiteman, Discretization error and modelling error in the context of the rapid inflation of hyperelastic membranes, IMA J. Numer. Anal. 30 (2010), 302-333.

21. S. Shaw and J. R. Whiteman, A posteriori error estimates for space-time finite element approximation of quasistatic hereditary linear viscoelasticity problems, Comput. Methods Appl. Mech. Engrg. 193 (2004), 5551-5572.

22. E. Svensson, Multigrid methods on adaptively refined triangulations: practical considerations, Department of Mathematical Sciences, Chalmers University of Technology and University of Gothenburg, Preprint 2006:23.

Department of Mathematics, University of Kurdistan, P. O. Box 416, Sanandaj, Iran

E-mail address: f.saedpanah@uok.ac.ir, f_saedpanah@yahoo.com 